\title{
Aberrant oligodendroglial LDL receptor orchestrates demyelination in chronic cerebral ischemia
}

\author{
Yi Xie, ${ }^{1}$ Xiaohao Zhang, ${ }^{1}$ Pengfei Xu, ${ }^{2}$ Nana Zhao, ${ }^{1}$ Ying Zhao, ${ }^{1}$ Yunzi Li, ${ }^{1}$ Ye Hong, ${ }^{1}$ Mengna Peng, ${ }^{1}$ Kang Yuan, ${ }^{3}$ Ting Wan, ${ }^{1}$ \\ Rui Sun, ${ }^{3}$ Deyan Chen, ${ }^{4}$ Lili Xu, ${ }^{5}$ Jingjing Chen, ${ }^{3}$ Hongquan Guo, ${ }^{6}$ Wanying Shan, ${ }^{1}$ Juanji Li, ${ }^{1}$ Rongrong Li, ${ }^{1}$ Yunyun Xiong, ${ }^{7,8}$ \\ Dezhi Liu, ${ }^{9}$ Yuhui Wang, ${ }^{10}$ Ceorge Liu, ${ }^{10}$ Ruidong Ye, ${ }^{1}$ and Xinfeng Liu ${ }^{1,2}$

\begin{abstract}
'Department of Neurology, Affiliated Jinling Hospital, Medical School of Nanjing University, Nanjing, Jiangsu, China. ${ }^{2}$ Stroke Center \& Department of Neurology, The Affiliated Hospital of USTC, Division of Life Sciences and Medicine, University of Science and Technology of China, Hefei, Anhui, China. ${ }^{3}$ Department of Neurology, Jinling Hospital, Nanjing Medical University, Nanjing, Jiangsu, China. ${ }^{4}$ Center for ${ }^{6}$ Department of Neurology, Jinling Hospital, Southern Medical University, Nanjing, liangsu, China. ${ }^{7}$ China National Clinical Research Center for Neurological Diseases, Beijing, China. ${ }^{8}$ Vascular Neurology,

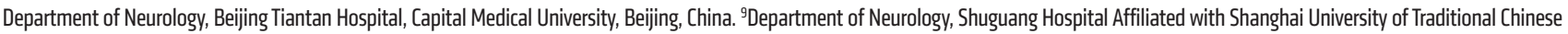 \\ Public Health Research, Medical School of Nanjing University, Nanjing, Jiangsu, China. ${ }^{5}$ Department of Neurology, Nanjing Brain Hospital Affiliated with Nanjing Medical University, Nanjing, Jiangsu, China. \\ Medicine, Shanghai, China. ${ }^{10}$ Institute of Cardiovascular Sciences, School of Basic Medicine, Peking University Health Science Center, Beijing, China.
}

\begin{abstract}
Oligodendrocytes express low-density lipoprotein receptor (LDLR) to endocytose cholesterol for the maintenance of adulthood myelination. However, the potential role of LDLR in chronic cerebral ischemia-related demyelination remains unclear. We used bilateral carotid artery stenosis (BCAS) to induce sustained cerebral ischemia in mice. This hypoxic-ischemic injury caused a remarkable decrease in oligodendroglial LDLR, with impaired oligodendroglial differentiation and survival. Oligodendroglial cholesterol levels, however, remained unchanged. Mouse miR-344e-3p and the human homolog miR-410-3p, 2 miRNAs directly targeting Ldlr, were identified in experimental and clinical leukoaraiosis and were thus implicated in the LDLR reduction. Lentiviral delivery of LDLR ameliorated demyelination following chronic cerebral ischemia. By contrast, LdIr ${ }^{1-}$ mice displayed inadequate myelination in the corpus callosum. Ldlr ${ }^{1-}$ oligodendrocyte progenitor cells (OPCs) exhibited reduced ability to differentiate and myelinate axons in vitro. Transplantation with Ldlr ${ }^{-1-}$ OPCs could not rescue the BCASinduced demyelination. Such LDLR-dependent myelin restoration might involve a physical interaction of the Asn-ProVal-Tyr (NPVY) motif with the phosphotyrosine binding domain of Shc, which subsequently activated the MEK/ERK pathway. Together, our findings demonstrate that the aberrant oligodendroglial LDLR in chronic cerebral ischemia impairs myelination through intracellular signal transduction. Preservation of oligodendroglial LDLR may provide a promising approach to treat ischemic demyelination.
\end{abstract}

\section{Introduction}

Oligodendrocytes, the only cells capable of forming myelin in white matter, are highly sensitive to hypoxic-ischemic injury $(1,2)$. Damage to oligodendrocytes triggers rapid and profound demyelination, which ultimately results in axonal degeneration $(3,4)$. This is associated with a high burden of neurobehavioral disorders and sensorimotor dysfunctions in patients (5). Thus, understanding the mechanism underlying ischemic demyelination is of crucial importance in clinical neurology. Oxidative stress, inflammation, and excitotoxicity have been put forward as underlying contributors to the initiation of oligodendrocyte injury under hypoxic-ischemic conditions since the last century $(1,5,6)$. However, mechanisms behind the cell-autonomous regulation of demyelination in ischemia are only beginning to be unraveled.

Authorship note: $\mathrm{Y} X \mathrm{Xie}, \mathrm{XZ}$, and PX contributed equally to this work. RY and X Liu are co-senior authors

Conflict of interest: The authors have declared that no conflict of interest exists. Copyright: () 2021, American Society for Clinical Investigation.

Submitted: October 8, 2019; Accepted: October 29, 2020; Published: January 4, 2021.

Reference information: J Clin Invest. 2021;131(1):e128114.

https://doi.org/10.1172/JCl128114.
Low-density lipoprotein receptors (LDLRs), enriched in oligodendrocytes, have been found to endocytose locally synthesized cholesterol. Cholesterol is integrated by oligodendrocytes into myelin sheaths and maintains normal myelination in adulthood (7). Hence, LDLRs may play an important role in the regulation of myelination/demyelination. Reduced LDLR levels have been linked with impaired oligodendrocyte differentiation (8). LDLR mutation-induced familial hypercholesterolemia has been found as a risk factor for white matter hyperintensities (9-11). However, the involvement of LDLR in myelination has been largely attributed to its regulation of cholesterol homeostasis. The signal transduction function of LDLR has scarcely been discussed. A clinical study reports that pravastatin cannot affect the progression of white matter lesions in elderly patients, though it reduced the LDL level to $34 \%$ of the baseline (12). Such discrepancy indicates that demyelinating disorders may involve local LDLR and downstream defects, rather than systemic LDLR-dependent cholesterol metabolism.

In this study, we investigated the potential role of LDLR in ischemic demyelination. A remarkable decrease in oligodendroglial LDLR expression was accompanied by white matter demyelination following bilateral carotid artery stenosis (BCAS). Mouse 
miR-344e and its human homolog miR-410-3p were potentiated in BCAS mice and in leukoaraiosis patients. They may account for the LDLR reduction through directly targeting $L d l r$ mRNA. The lentiviral supplementation of LDLR ameliorated the demyelination following BCAS. LDLR deficiency impaired oligodendroglial differentiation and myelination, which could also be rescued with the reexpression of LDLR. Transplantation with $\mathrm{Ldlr}^{-1}$ oligodendrocyte progenitor cells (OPCs) could not attenuate BCASinduced demyelination. Such LDLR-dependent restoration of myelination may involve the Asn-Pro-Val-Tyr (NPVY) motif with subsequent interaction with the phosphotyrosine binding (PTB) domain of Shc. These findings, collectively, reveal a critical role of LDLR in the regulation of oligodendroglial function and demyelination following chronic cerebral ischemia.

\section{Results}

Demyelination persists in the corpus callosum following chronic cerebral ischemia. BCAS induced a typical cerebral hemodynamic insufficiency, as previously reported (13) (Figure 1A). Using histochemical evaluations, we found that the integrity of myelin was gradually compromised in the corpus callosum (CC) after 4 to 8 weeks of BCAS (Figure 1, B and C; $P=0.014$ and 0.001). Myelinated fibers, as defined by myelin basic protein (MBP) and neurofilament (NF200) expression, were significantly reduced (Figure $1, \mathrm{D}-\mathrm{F})$. Of particular interest, the loss of MBP immunoreactivity persisted 8 weeks after BCAS when immunofluorescence was normalized to that of NF200 (Figure 1G; $P=0.015$ ). Electron microscopy (EM) provided direct evidence for demyelination with sustained decreases in myelinated axons and myelin thickness following chronic cerebral ischemia (Figure $1, \mathrm{H}-\mathrm{K}$ ). BCAS mice at 4 weeks and 8 weeks achieved a respective decrease of $26.7 \%$ and $47.1 \%$ in the numbers of total axons $(P=0.001$ and $P<0.001)$, but had a decrease of $57.8 \%$ and $96.9 \%$, respectively, in the myelinated axons (both $P<0.001$ ), indicating that BCAS-related demyelination was more severe than the axonal loss. Consistently, MBP and myelin proteolipid protein (PLP) were significantly decreased at the protein level 8 weeks after the insult (Figure 1, L-N).

Hypoxic-ischemic injury disturbs OPC proliferation and differentiation. OPC proliferation, as defined by the incidence of platelet-derived growth factor receptor alpha-positive (PDGFR- $\alpha-$ positive) and Ki67-positive cells, was increased at 4 weeks of BCAS compared with controls (Figure $2, \mathrm{~A}$ and $\mathrm{B} ; P<0.001$ ), followed by a substantial decrease at 8 weeks compared with BCAS at 4 weeks (Figure 2, A and B; $P<0.001$ ). In normoxic differentiation medium, OPCs readily differentiated into $\mathrm{MBP}^{+}$oligodendrocytes with highly developed ramified processes. Nevertheless, exposure to $\mathrm{CoCl}_{2}$ or oxygen glucose deprivation (OGD) resulted in a decrease in the number of $\mathrm{MBP}^{+}$cells and the complexity of OPC processes compared with controls (Figure 2, C-E; both $P$ $<0.001)$. A consistent decrease was revealed in the expression levels of mature oligodendrocyte markers, MBP and 2',3'-cyclic nucleotide 3'-phosphodiesterase (CNPase) (Figure 2, F and G). Increased level of hypoxia-inducible factor 1-alpha (HIF-1 $\alpha$ ) compared with controls confirmed hypoxic conditions (Figure 2, F and $G ; P=0.002$ and 0.009 , respectively).

Hypoxic-ischemic injury reduces the survival of oligodendrocytes. We next asked whether demyelinating injury during chronic isch- emia was associated with the loss of mature oligodendrocytes. There were significant reductions in adenomatous polyposis coli protein clone $\mathrm{CC} 1$ expression by oligodendrocyte transcription factor 2-expressing (Olig2-expressing) cells following BCAS (Figure 2, $\mathrm{H}$ and I). The $\mathrm{CC}^{+}$mature oligodendrocytes were arrested along with the hypoperfusion $(F=163.342, P<0.001)$. Furthermore, we found more TUNEL-positive oligodendroglial cells in the CC of BCAS mouse brains (Supplemental Figure 1, A and B; supplemental material available online with this article; https:// doi.org/10.1172/JCI128114DS1). After exposure to $\mathrm{CoCl}_{2}$ or OGD, oligodendrocytes had robust TUNEL signal, apoptosis-inducing factor (AIF) and light chain 3 beta (LC3- $\beta$ ) labeling, and marked nuclear translocation of AIF and LC3- $\beta$ (Supplemental Figure 1, C and D). These results indicated that hypoxia may initiate substantial oligodendroglial death, including apoptosis, parthanatos, and autophagy, and result in subsequent myelin malformation.

Oligodendroglial LDLR is abrogated with unchanged cholesterol levels. As oligodendroglial LDLR is of great importance during adult myelin synthesis and remodeling (7), we then probed LDLR expression in BCAS mice. LDLR was highly expressed in OPCs and mature oligodendrocytes compared with other neural cells (Supplemental Figure 2). In the CC of mice subjected to BCAS, Ldlr mRNA levels decreased $67.5 \%$ to $72.6 \%$ after 4 to 8 weeks compared with controls (Figure 3A; $P=0.004$ and 0.003). LDLR protein levels accordingly dropped to $27.1 \%$ and $28.1 \%$ in 4 -week BCAS and 8-week BCAS mice compared with controls (Figure 3, $\mathrm{B}$ and $\mathrm{C}$; both $P=0.002$ ). Cells positive for both CC1 and LDLR revealed a dramatic loss of LDLR in oligodendrocytes, which may account for the LDLR reduction in the CC following BCAS (Figure 3, D-F). As for oligodendrocytes differentiated in hypoxic medium, similar decreases were observed in the LDLR mRNA and protein levels (Figure 3, G-I; mRNA, $t=4.844, P=0.040$; protein, $t=5.856, P=0.028)$. Immunofluorescence analysis showed that oligodendroglial LDLR was decreased to less than half of that in cells cultured in normal medium (Figure 3, J-L).

It is known that LDLR is a ubiquitously expressed key receptor for maintaining cholesterol homeostasis (14). We further explored whether cholesterol content was altered following the reduction in LDLR levels. Interestingly, after normalizing to brain weight, no significant changes were detected in cholesterol levels of the CC in BCAS mice (Figure 3M). Correspondingly, cultured oligodendrocytes subjected to $\mathrm{CoCl}_{2}$ or OGD exhibited no changes in cellular cholesterol level and LDL uptake (Figure 3, $\mathrm{N}$ and $\mathrm{O}$ ).

miR-344e/410-3p is induced in experimental and clinical leukoaraiosis. To study $L d l$ r regulation at the miRNA level, high-throughput miRNA sequencing was performed. The CC of 4-week BCAS and 8-week BCAS mice mapped to 1915 known mouse miRNA sequences. Among them, 175 miRNAs were found altered, with a fold change of 2 or greater (Supplemental Figure 3, A and B) and 56 were upregulated at both 4 and 8 weeks after BCAS (Supplemental Figure 3C). We further intersected the co-upregulated 56 miRNAs with predicted miRNAs in TargetScan (http://www. targetscan.org/vert_72/) andidentified13miRNAsthatpotentially target $L d l r$ (Figure $4 \mathrm{~A})$. Following real-time polymerase chain reaction (PCR) validation, $\mathrm{miR}-344 \mathrm{e}-3 \mathrm{p}$ was detected at 6.1-fold and 6.6-fold higher levels in the CC of 4-week BCAS and 8-week 
A

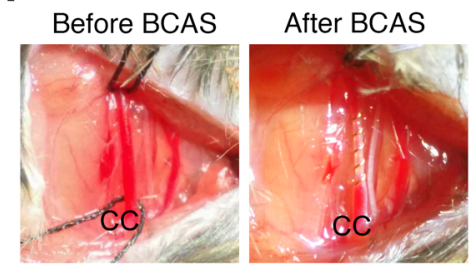

B

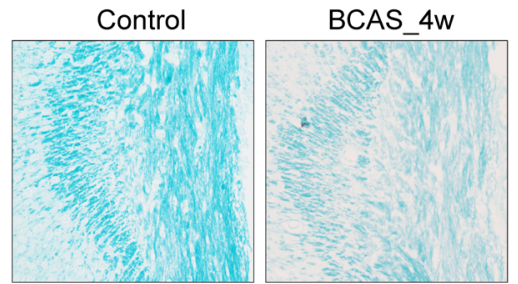

D
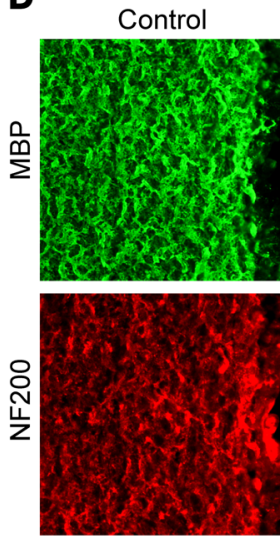

H

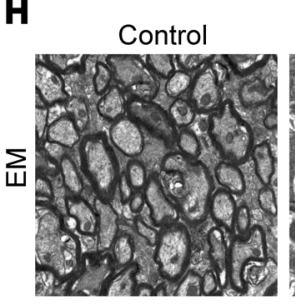

J

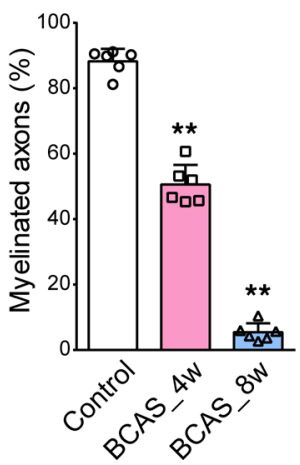

$\mathbf{K}$

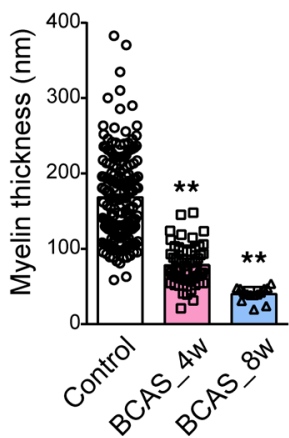

E
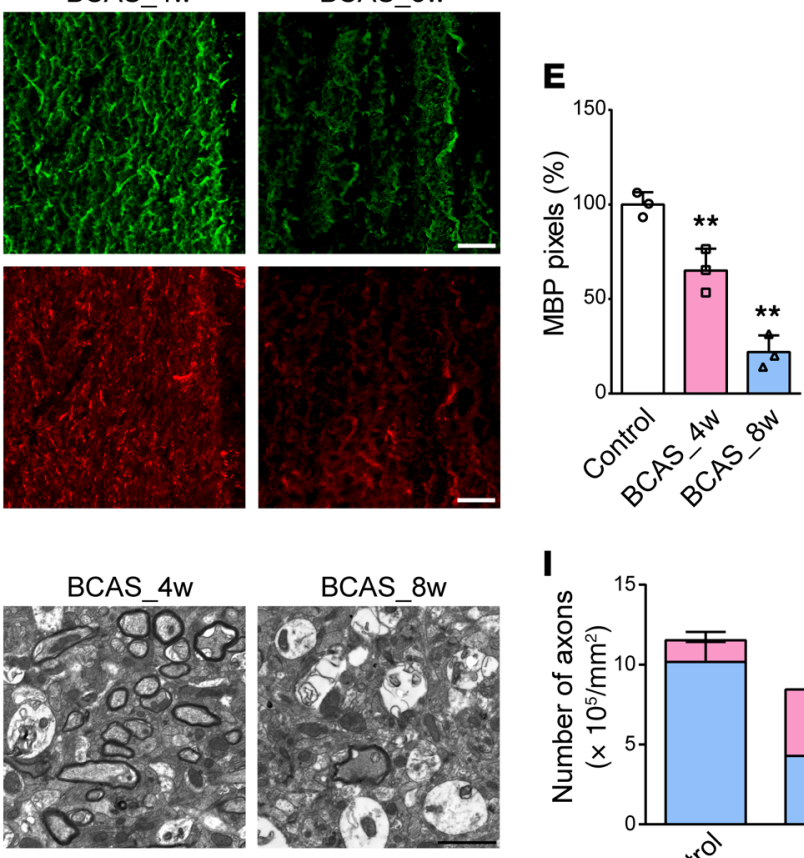

BCAS_8w
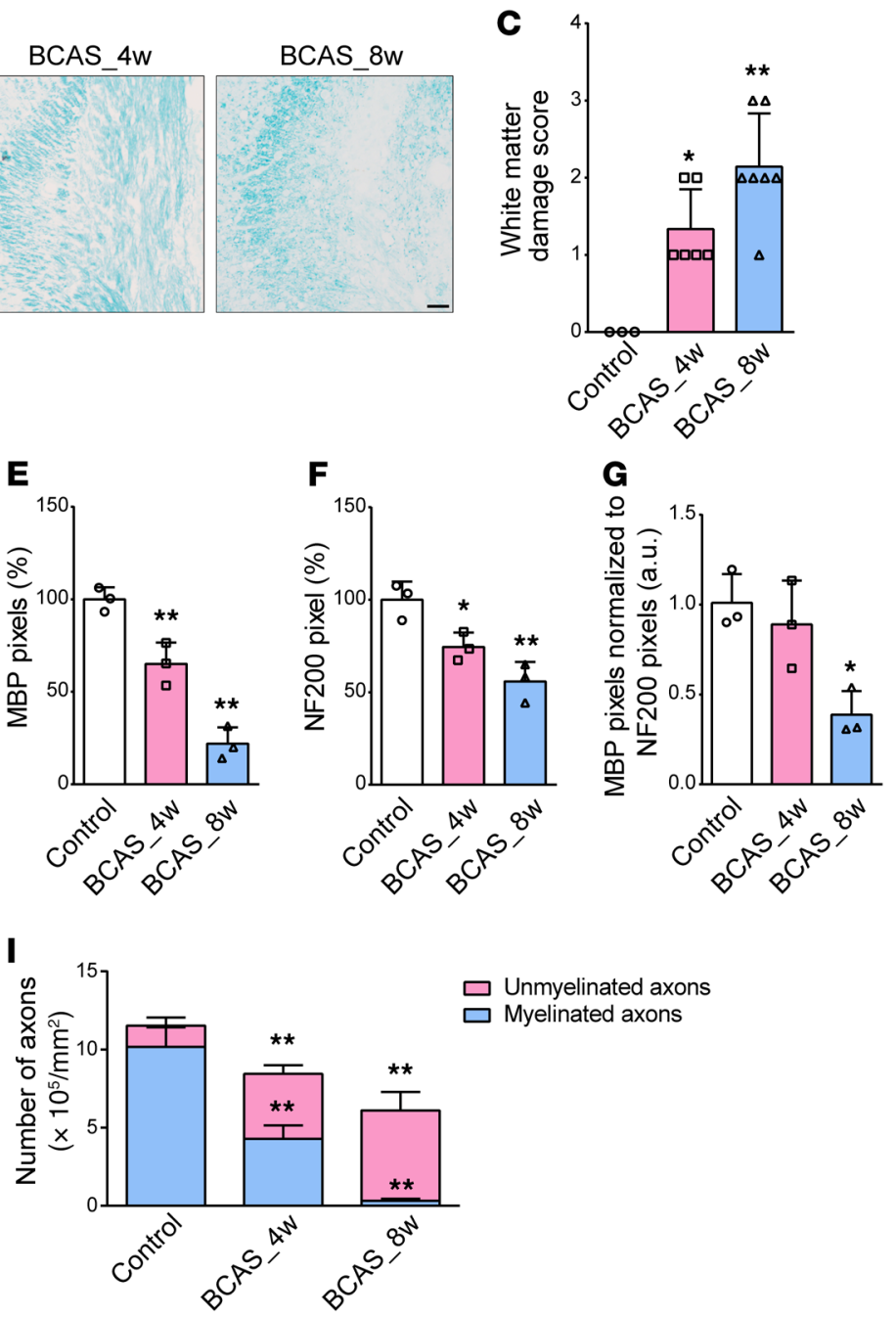

Figure 1. Pathological changes to cerebral myelination after BCAS. (A) A microcoil with an inner diameter of 0.18 mm was twined by rotating it around the CC. (B and C) Representative Luxol fast blue staining and quantitative analysis of white matter damage severity in the CC from sham, 4-week BCAS (BCAS_4w), and BCAS_8w mice ( $n \geq 3$ in each group). (D-G) Representative MBP and NF200 immunostaining in the CC and their pixel ratios, which were calculated as the numbers of MBP or NF200 pixels divided by the total numbers of pixels within the field of view ( $n=3$ in each group). (H-K) Representative EM images from control and BCAS mice and statistical analyses of myelinated axons and myelin thickness ( $n=6$ in each group). ( $\mathbf{L}-\mathbf{N}$ ) Immunoblotting analyses of MBP and PLP expression in the CC of sham and BCAS mice. $\beta$-Actin protein served as control ( $n=3$ in each group). All data presented as mean $\pm \mathrm{SD}$. ${ }^{*} P<0.05,{ }^{*} P<0.01$ vs. control by 1-way ANOVA with Tukey's post hoc test. Scale bars: $20 \mu \mathrm{m}$ (B and $\left.\mathbf{D}\right)$ and $2 \mu \mathrm{m}$ (H). 

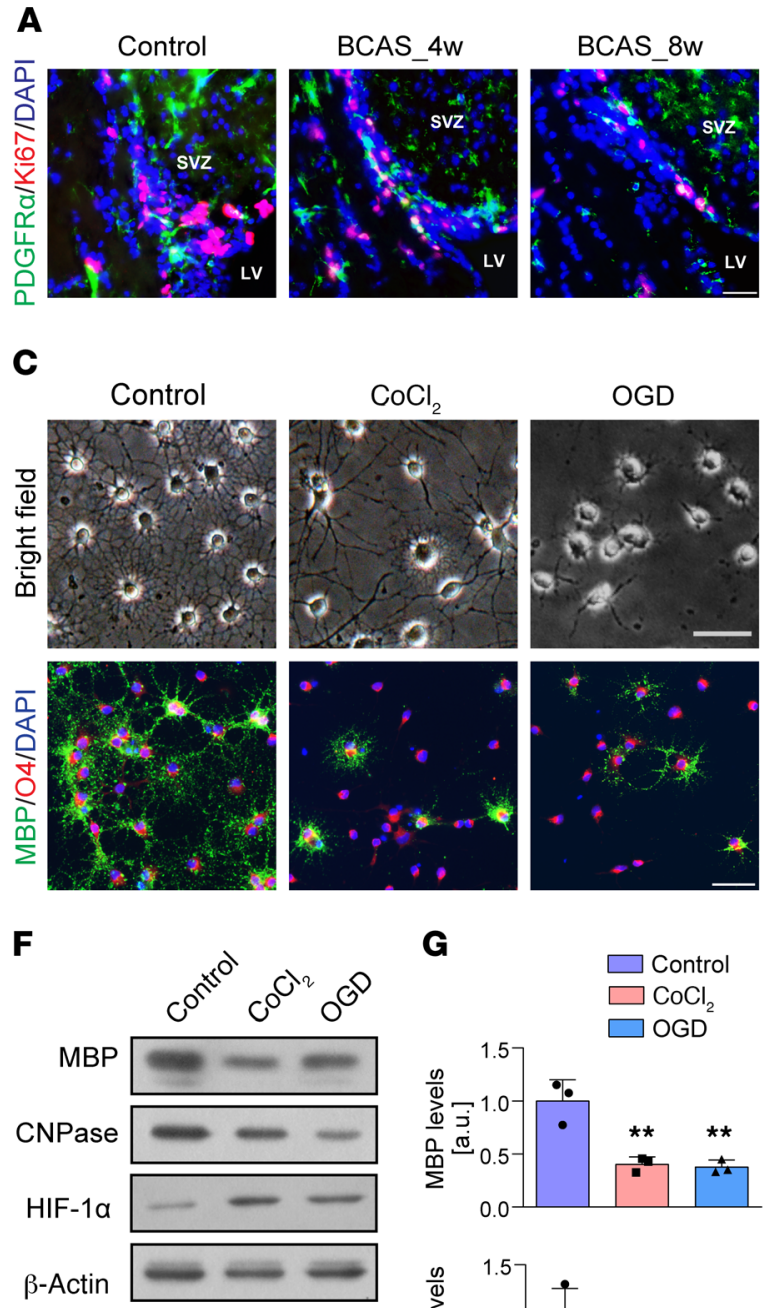

G

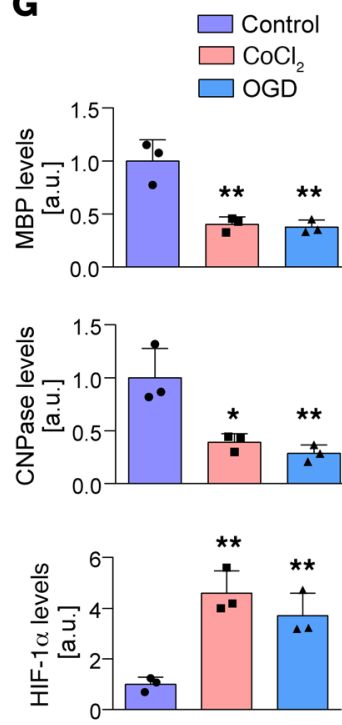

B
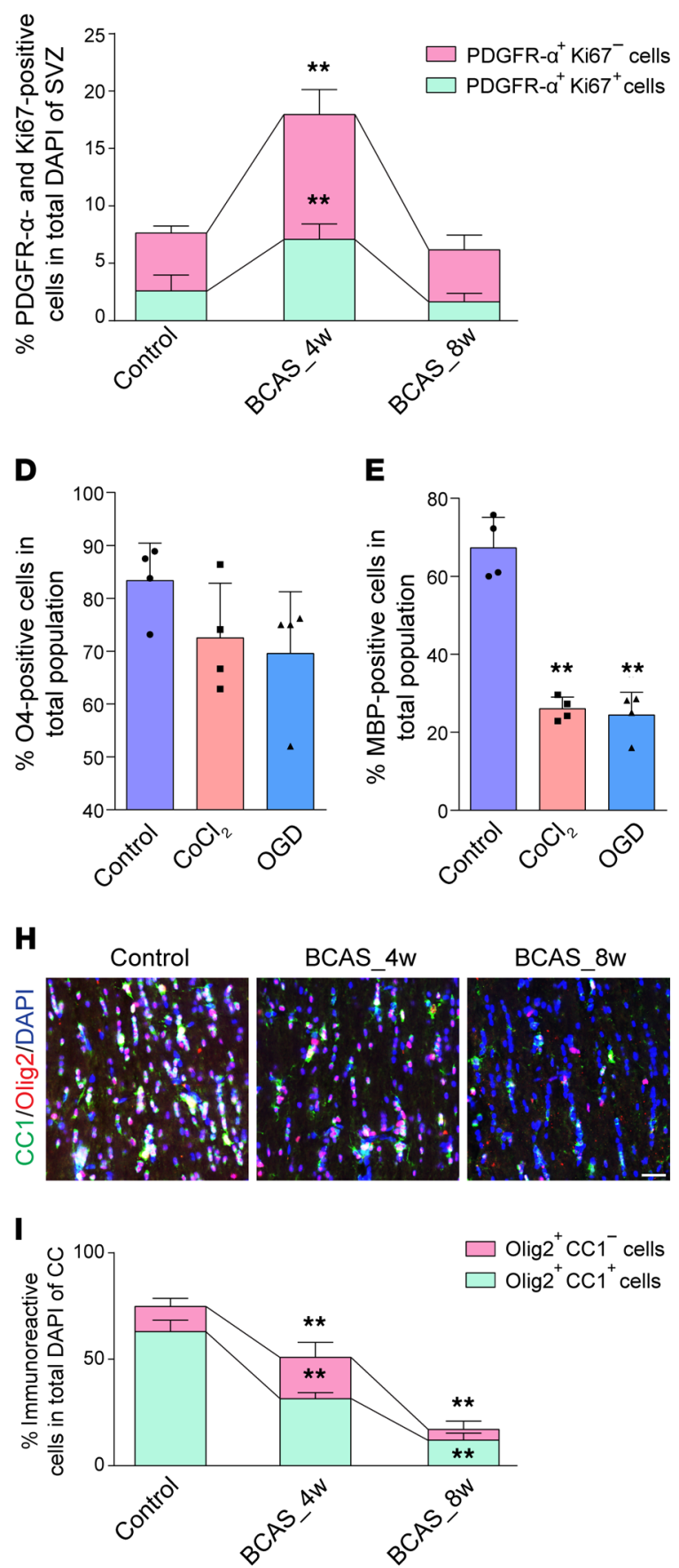

Figure 2. Pathological changes to OPCs and mature oligodendrocytes after hypoxic-ischemic injury. (A and $\mathbf{B}$ ) Changes in endogenous OPC numbers and their proliferation represented by Ki67+PDGFR- $\alpha^{+}$cells as a percentage of total DAPI ${ }^{+}$cells in the subventricular zone (SVZ) ( $n=4$ in each group). (C-E) Light microscopic appearance and fluorescence images displaying the differences in OPC differentiation and its quantification ( $n=4$ experiments). (F and G) Immunoblotting analyses of MBP and CNPase expression showed that hypoxia induced by $\mathrm{CoCl}_{2}(10 \mu \mathrm{M})$ or OCD ( 2 hours) impairs OPC maturation ( $n=3$ experiments). (H and I) Immunofluorescence images and quantification of the numbers of CC1+Olig2 ${ }^{+}$cells in the CC of BCAS mice compared with controls ( $n=3$ in each group). Data are presented as mean \pm SD. ${ }^{*} P<0.05,{ }^{* *} P<0.01$ vs. controls by 1 -way ANOVA with Tukey's post hoc test. Scale bars: $20 \mu \mathrm{m}$.

BCAS mice compared with control (Figure 4B; $P=0.004$ and 0.002 , respectively), the expression profiles of which were consistent with the reductions in tissue levels of LDLR. To define the cell-autonomous nature of miR-344e-3p during chronic ischemia, we applied miRNA fluorescent in situ hybridization (FISH) and noted a substantial increase in miR-344e-3p in mature oligoden- drocytes in BCAS mouse brains (Figure 4C). We then examined the expression of those increased miRNAs in $\mathrm{CoCl}_{2}$-treated rat oligodendrocytes. Ten miRNAs were identified as sharing the same seed region sequences with the 13 mouse miRNAs (Supplemental Table 1). Oligodendroglial miR-410-3p, analogous to mouse miR-344e-3p, had the highest increase of 5.9-fold when 
A

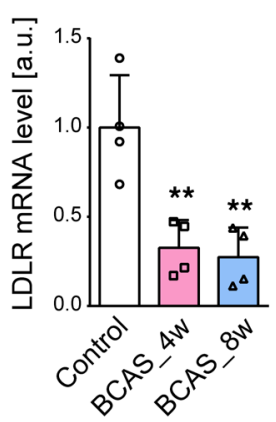

E

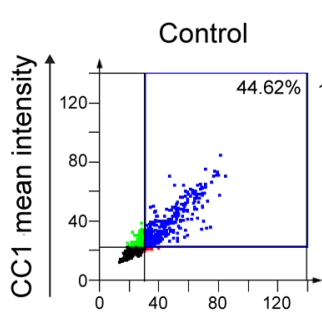

B

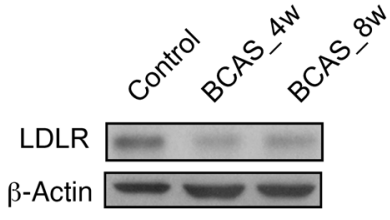

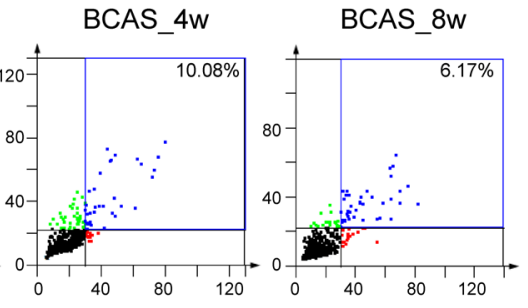

LDLR mean intensity
C

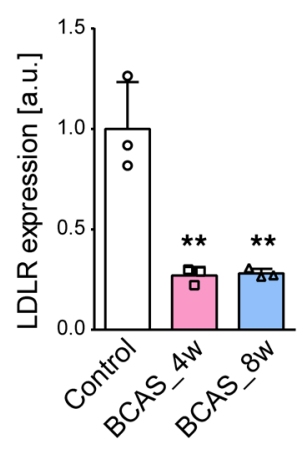

$\mathbf{F}$

D

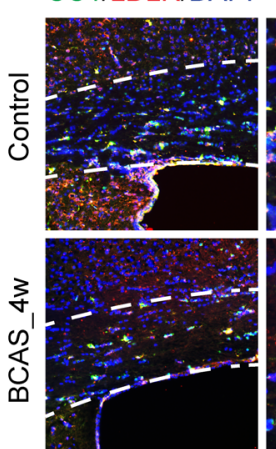

Enlargement
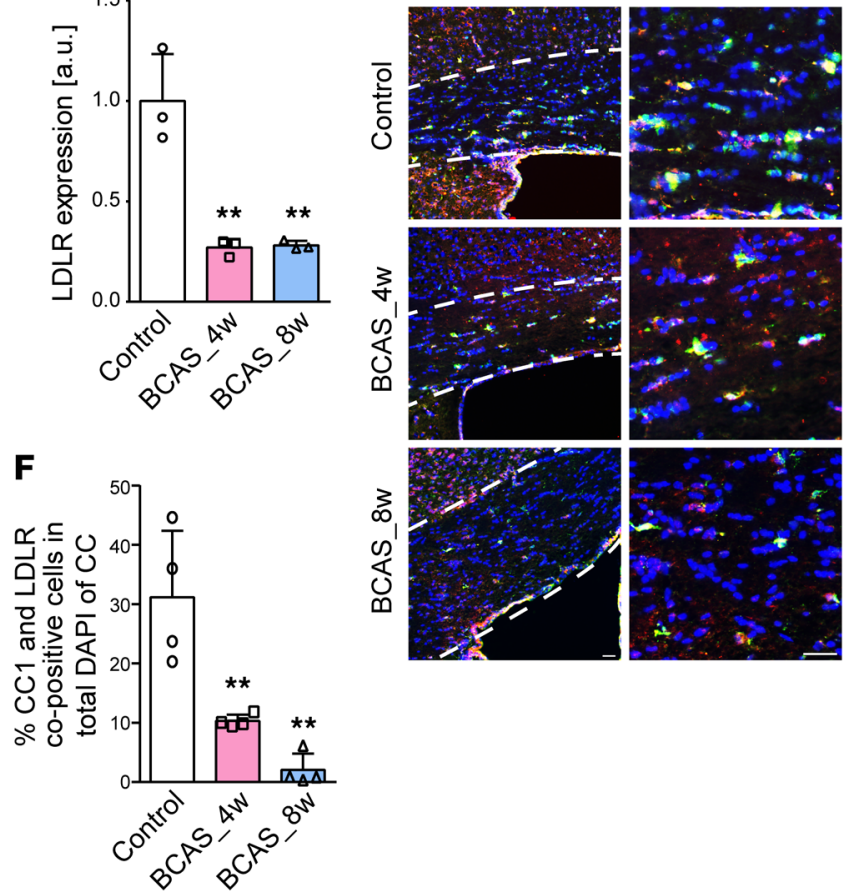

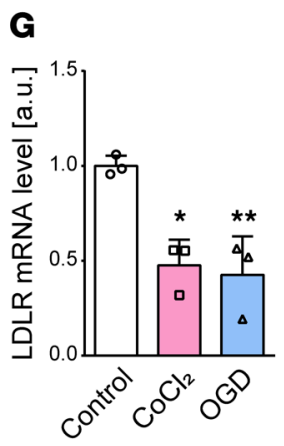

H

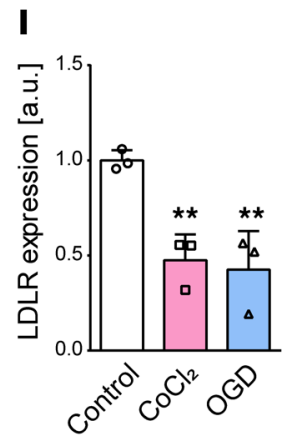

J
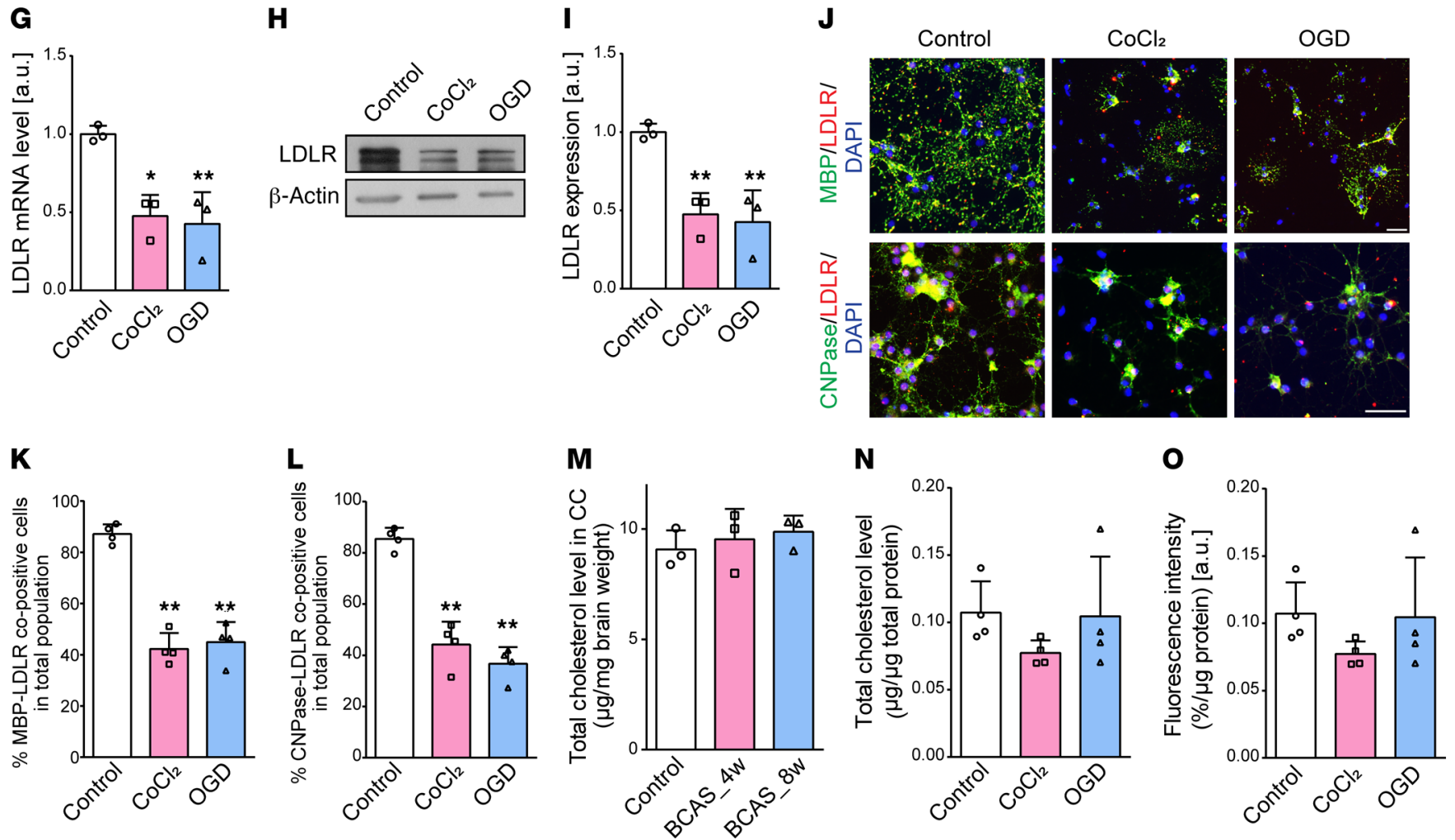

Figure 3. Hypoxic-ischemic injury compromises oligodendroglial LDLR expression without affecting cholesterol levels. (A-C) LDLR mRNA and protein levels in the CC of mice ( $n \geq 3$ in each group). (D-F) Immunofluorescence images and quantification showing the changes in LDLR (red) expression of CC ${ }^{+}$ (green) oligodendrocytes in BCAS mice ( $n=4$ in each group). The CC boundary is indicated by the white dashed line. (G-I) Oligodendroglial LDLR mRNA and protein expression ( $n=3$ experiments). (J-L) Immunofluorescence images and quantification showing the changes in LDLR (red) expression of in vitro mature oligodendrocytes (MBP+CNPase ${ }^{+}$, green) in control, $\mathrm{COCl}_{2}$, and $\mathrm{OCD}$ groups ( $n=4$ experiments). (M-0) Quantification of cholesterol content of tissues and cells, as well as the oligodendroglial ability to take up LDL ( $n \geq 3$ experiments in each group). Data presented as mean \pm SD. ${ }^{*} P<0.05$, ${ }^{* *} P<0.01$ vs. controls by 1-way ANOVA with Tukey's post hoc test. Scale bars: $20 \mu \mathrm{m}$. 


\section{A}
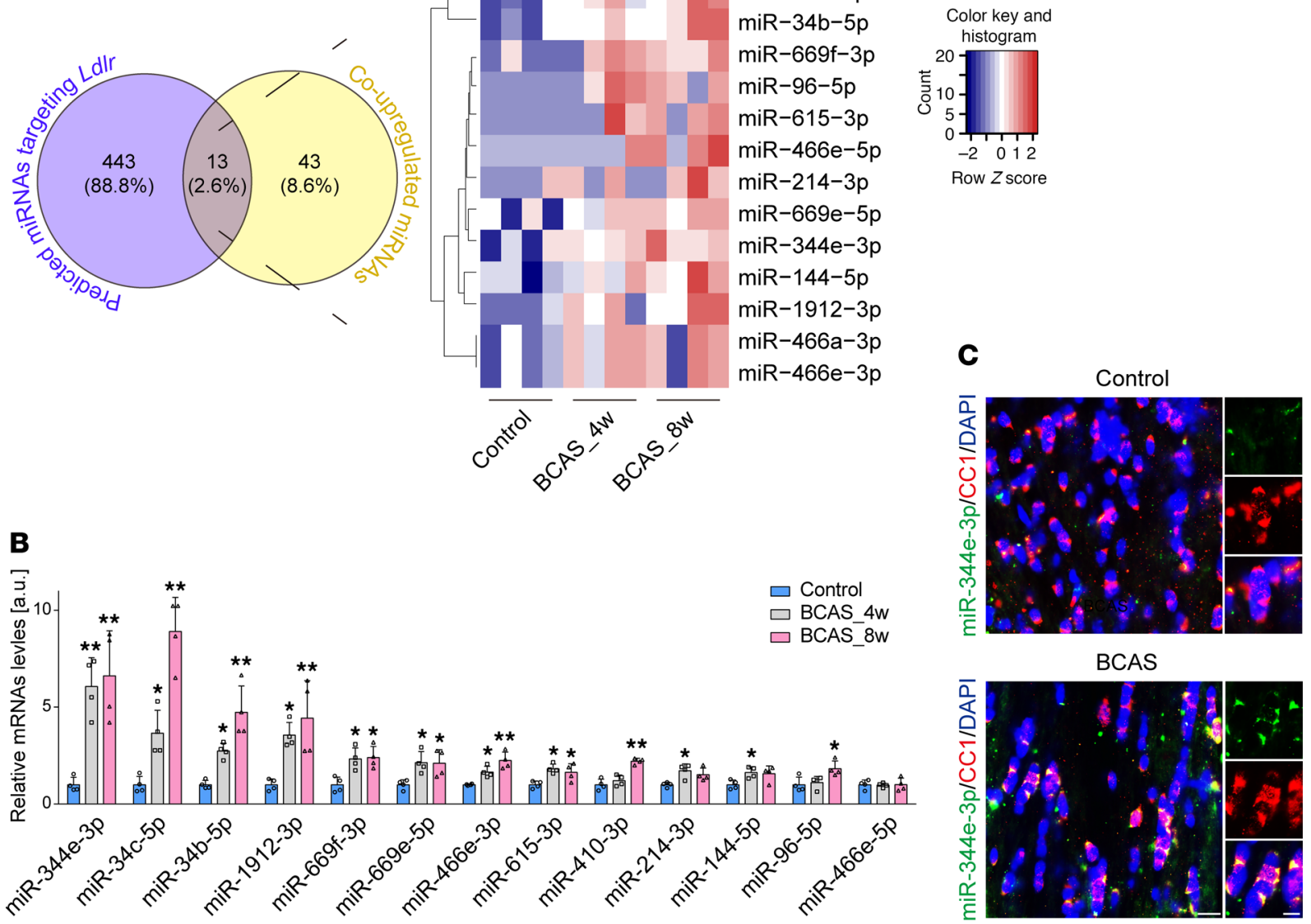

D

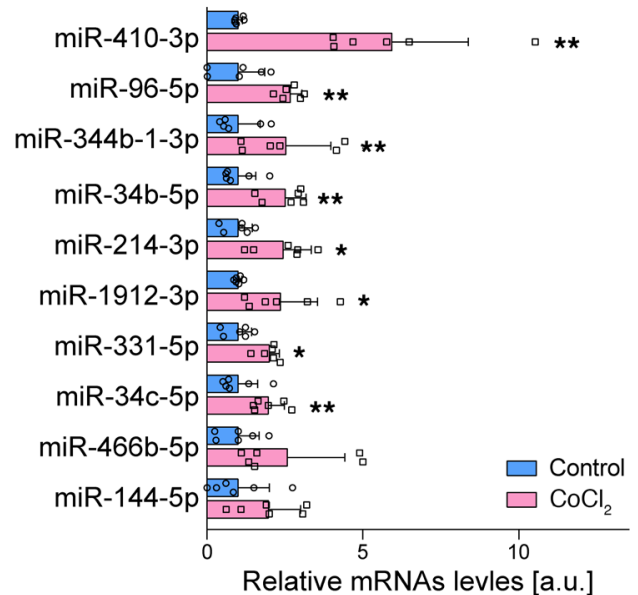

\section{$\mathbf{E}$}

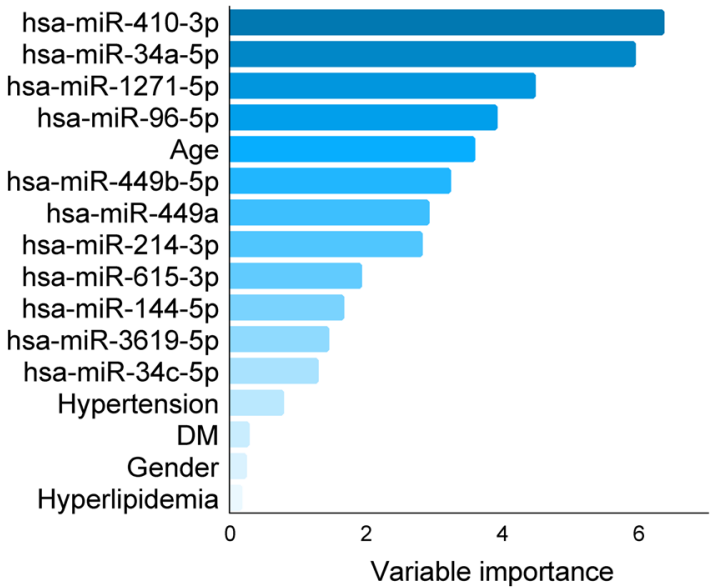

Figure 4. Oligodendrocyte-originated miR-344e/410-3p is identified as a specific signature in ischemic white matter injury. (A) Systematic screening of the upregulated miRNAs targeting LDLR after BCAS ( $n=4$ in each group). (B) Real-time PCR for validation of 13 increased miRNAs in the CC of BCAS mice ( $n=4$ in each group). (C) Combined FISH and immunofluorescence for miR-344e-3p (green) and CC1+ (red) mature oligodendrocytes in BCAS mice. (D) Realtime PCR for validation of 10 increased rat miRNAs in cultured oligodendrocytes treated with $\mathrm{CoCl}_{2}$ ( $n=6$ experiments). (E) Random-forest analysis of the factors contributing to leukoaraiosis diagnosis. DM, diabetes mellitus. Data presented as mean \pm SD. ${ }^{*} P<0.05$, ${ }^{* *} P<0.01$ vs. controls by 1 -way ANOVA with Tukey's post hoc test (B) or paired $t$ test (D). Scale bars: $10 \mu \mathrm{m}$.

cells were chronically treated with $\mathrm{CoCl}_{2}$ compared with control (Figure 4D; $P=0.0046)$.

We continued to evaluate the clinical significance of these miRNAs. We prospectively recruited 118 patients with leuko- araiosis (mean age, $67.0 \pm 9.9$ years; $64.4 \%$ male) and 69 subjects without leukoaraiosis (mean age, $60.5 \pm 6.5$ years; $58.0 \%$ male). Eleven human miRNAs were selected for possessing the seed sequence in common with the above-mentioned 13 mouse 
miRNAs (Supplemental Table 1). Univariate logistic regression analysis demonstrated that advanced age, hypertension, diabetes mellitus, and increased log-transformed serum levels of hsamiR-410-3p, hsa-miR-34a-5p, hsa-miR-1271-5p, hsa-miR-96-5p, hsa-miR-449b-5p, hsa-miR-449a, and hsa-miR-144-5p were associated with leukoaraiosis (Supplemental Table 2 and Supplemental Figure 4). Furthermore, log-transformed hsa-miR410-3p (odds ratio [OR] 5.756; 95\% confidence interval [CI] 2.069-16.010; $P=0.001)$ and hsa-miR-34a-5p (OR, 3.733; 95\% CI, 1.032-13.507; $P=0.045$ ) were identified as independent risk factors for leukoaraiosis in the multivariate logistic regression analysis (Supplemental Table 2). Variable selection using random-forest analysis identified miR-410-3p as the most valuable contributor to leukoaraiosis (Figure 4E).

$L d l r$ is a direct target of $m i R-344 e / 410-3 p$ in oligodendrocytes. The mouse miR-344e-3p and human homolog miR-410-3p share a conserved predicted binding site in the $3^{\prime}$ untranslated region (UTR) of $L d l r$ (Figure 5A and Supplemental Table 3). We conducted luciferase reporter assays in 293T cells to ascertain whether miR-344e/410-3p could specifically recognize the 3' UTR of Ldlr. As revealed in Figure 5B, transfection of miR$344 \mathrm{e} / 410-3 \mathrm{p}$ mimics substantially inhibited the activity of the Ldlr 3' UTR compared with negative control-transfected cells (miR-344e-3p, $t=11.857, P<0.001 ;$ miR-410-3p, $t=18.842, P$ $<0.001)$. In contrast, when we mutated the miR-344e/410-3p binding sites in the $3^{\prime}$ UTR of mouse and human $L d l r$, luciferase activities were significantly derepressed.

To determine whether miR-344e/410-3p affects endogenous levels of LDLR, we evaluated the mRNA and protein expression of LDLR in the oligodendrocytes treated with miR-410-3p agomir and antagomir. A robust uptake of Cy5-labeled miRNA was detected in the OPCs (Figure 5C). Transfection with miR-410-3p agomir significantly lowered LDLR mRNA and protein levels in the OPCs (Figure 5, D-F). Moreover, miR-410-3p agomir significantly inhibited OPC differentiation and myelin protein levels (Figure 5, G-K). However, although treatment with the miR-410$3 p$ antagomir induced a 1.9-fold higher expression of LDLR, it did not exert noticeable effects on OPC differentiation (Figure $5, \mathrm{G}-\mathrm{K})$, implying redundant oligodendroglial LDLR may not induce excessive myelination.

Overexpression of LDLR with NPVY motif preserves oligodendroglial differentiation and survival in the hypoxic condition. We employed genetic approaches to increase LDLR expression via lentiviral delivery of full-length LDLR directly into the OPC proliferation medium 3 days before incubation in $\mathrm{CoCl}_{2}$-treated differentiation medium (Figure 6A). GFP staining results illustrated a high $L d l r$-GFP transfection efficiency of $65.7 \%$ in vitro (Figure 6, B and C). OPC infection with lentivirus expressing LDLR revealed up to a 2.3-fold increase in LDLR expression (Figure 6, D and E). OPCs transfected with $L d l r$-GFP reporter virus demonstrated increased levels of MBP and CNPase expression compared with control lentivirus (Figure 6, D, F, and $G ; P=0.001$ and 0.003 ), which was further supported by an improved capability to differentiate and form myelin (Figure 6, $\mathrm{H}$ and I). Moreover, LDLR supplementation inhibited $\mathrm{CoCl}_{2}-$ induced oligodendroglial death, as assessed by TUNEL, AIF, and LC3- $\beta$ staining (Figure 6, J and K).
The NPxY motif, which is shared by most members of the LDLR family, has recently been shown to be important in OPC differentiation (15). We therefore hypothesized that the intracellular NPVY motif might be involved in ischemic demyelination. To test this possibility, we compared the effects between LDLR with WT NPVY and that with mutated NPVY, which was changed to Ala-Ala-Val-Ala (AAVA). Consistently, mutated LDLR was delivered into OPCs 3 days before $\mathrm{CoCl}_{2}$-induced differentiation (Figure 6A). Transfection efficiency was as high as $63.8 \%$ (Figure 6, $\mathrm{B}$ and $\mathrm{C}$ ). LDLR expression was increased to 2.5 -fold higher than that of control (Figure 6, D and E). Mutated LDLR-incubated oligodendrocytes more weakly expressed markers of mature oligodendrocytes, MBP and CNPase (Figure 6, D, F, and G). A markedly immature appearance was observed in mutated $L d l r$ GFP-infected oligodendrocytes (Figure 6, H and I). In addition, $\mathrm{CoCl}_{2}$-induced cellular death was not reversed by NPVY mutation (Figure 6, J and $\mathrm{K}$ ). Together, these data suggest that the NPVY motif is indispensable for the salutary effects of LDLR in hypoxia-related demyelination.

NPVY motif is required for the LDLR-induced attenuation of demyelination in vivo. We then asked whether in vivo genetic supplementation of LDLR could attenuate BCAS-induced demyelination and the role of the NPVY motif. To this end, WT or mutated full-length LDLR was stereotaxically injected into the CC of mice 2 days before BCAS surgery (Figure 7A).

First, we identified the cell specificity of the lentivirus manipulating LDLR expression in the CC. Robust GFP signals were captured within mature oligodendrocytes with successfully enhanced LDLR expression (Figure 7B). WT Ldlr-GFP virustreated mice achieved higher yields of endogenous proliferated OPCs and glutathione-S-transferase pi-positive (GST-pipositive) mature oligodendrocytes (Figure 7, C-E). The myelinrelated proteins, MBP, PLP, and myelin-associated glycoprotein (MAG), were maintained by WT LDLR (Figure 7, F-I). Compared with mice receiving the null GFP reporter virus, mice injected with lentiviral vectors encoding $L d l r$ displayed less white matter damage and higher percentages of myelinated axons with thicker myelin sheaths (Figure 7, J-M). On the other hand, mutated LDLR transfection did not exert a parallel preservation of myelin proteins and myelin sheath microstructures (Figure 7, F-M). WT LDLR-induced protection persisted at least 8 weeks following BCAS insult (Supplemental Figure 5).

Shc/MEK/ERK pathway mediates the subsequent cellular signaling of the LDLR NPVY motif. A previous study demonstrated that the NPxY domain in the LDLR-related proteins (LRPs) can provide docking sites for adaptor proteins, such as Shc (16). Thus, we conducted coimmunoprecipitation assays to investigate whether there is a physical association between LDLR and Shc. Immunoassay of lysates of brain tissue after immunoprecipitation with LDLR, analyzed by immunoblotting with anti-Shc antibody, revealed that LDLR can bind to the $66 \mathrm{kDa}$ isoform of Shc (Figure 8A). Next, we constructed fusion proteins containing WT and NPVY mutant LDLR and histidine (HIS), and another construct containing the PTB domain of Shc and GST (Figure 8B). A direct binding was uncovered between the NPVY motif and the PTB domain (Figure $8 C$ ). This interaction was not seen with the negative controls or with the mutated AAVA motif. 
A

mmu-miR-344e-3p 3'-UAUCAGUCCGAAACCAAUAUAG-5' hsa-miR-410-3p 3'-UGUCCGGUAGACACAAUAUAA-5'

Ldir 3' UTR WT

LdIr 3' UTR MUT
5'-...UGUUUUA I I I I

5'-...UGUUAAUAUAA...-3'

\section{B}
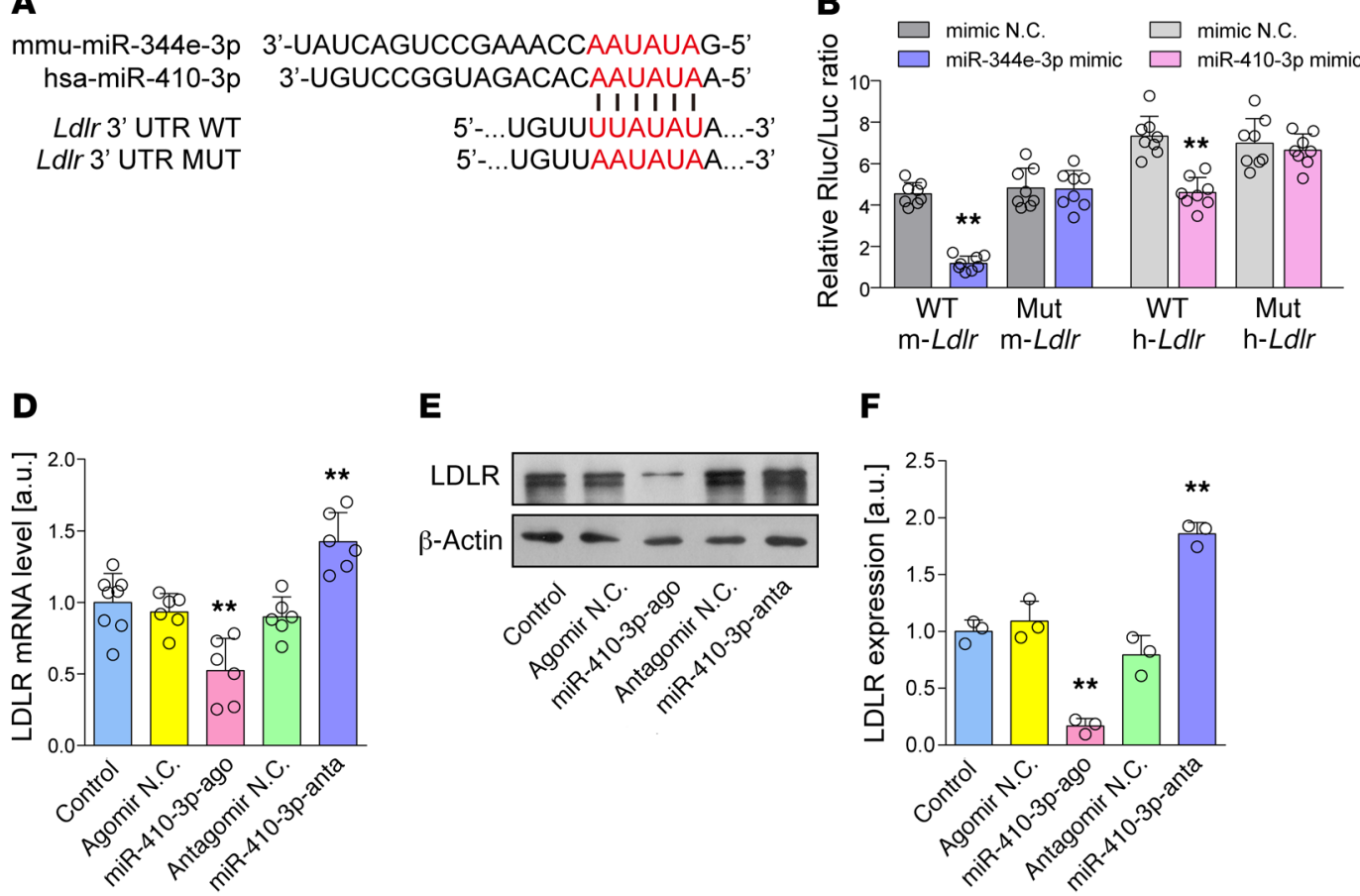

E

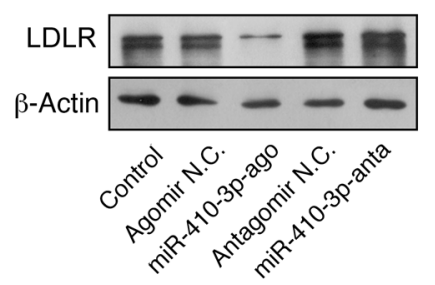

$\mathbf{F}$

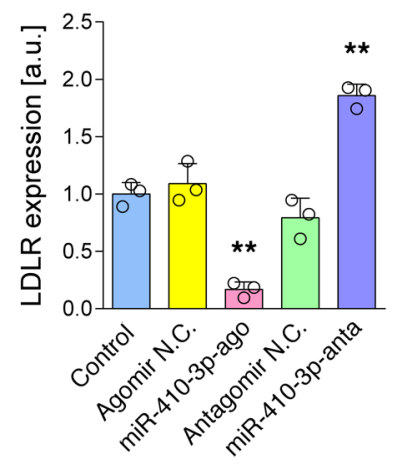

C NG2/Olig2/Cy5

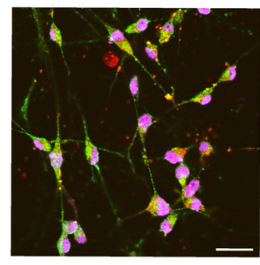

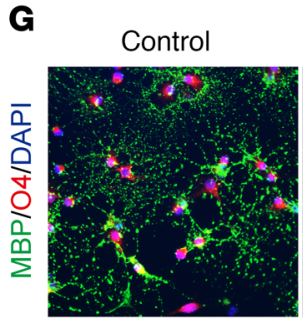

H

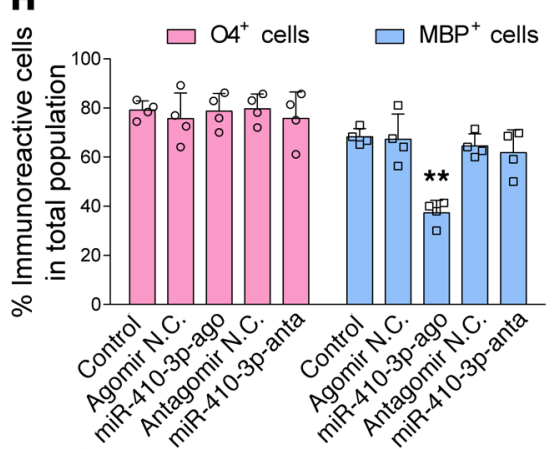

Agomir N.C.

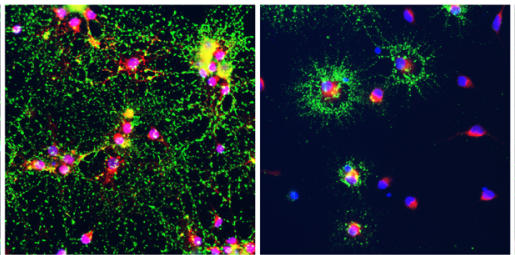

I

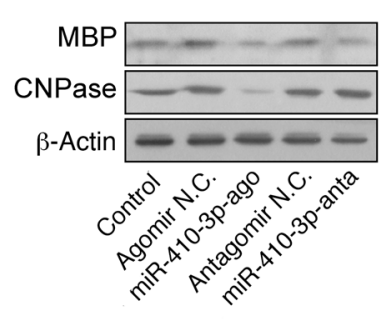

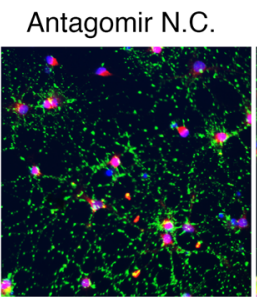

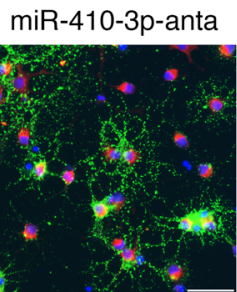

\section{$\mathbf{J}$}

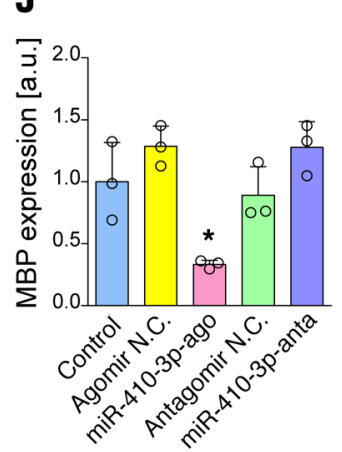

$\mathbf{K}$

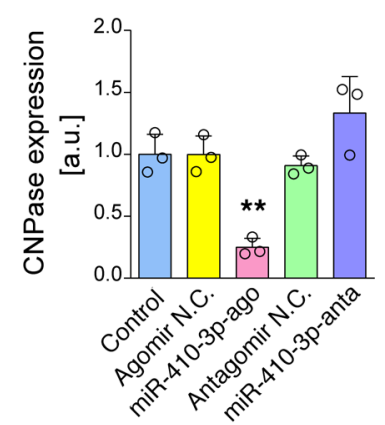

Figure 5. miR-344e/410-3p regulates LdIr expression and cell differentiation in OPCs. (A and B) Dual-luciferase reporter assay of the relationship between miR-344e/410-3p mimic and WT 3' UTR of mouse/human Ldlr ( $n=8$ experiments). N.C., negative control. (C) Uptake of miR-410-3p agomir or antagomir in cultured OPCs. NG2, neuron-glial antigen 2. (D-F) The endogenous expression of LDLR mRNA and protein after treatment with miR-410-3p agomir and antagomir ( $n \geq 3$ experiments). ( $\mathbf{G}$ and $\mathbf{H}$ ) Representative immunofluorescence images and quantification showing the mature MBP+ oligodendrocytes differentiated from OPCs after treatment with miR-410-3p agomir or antagomir ( $n=4$ experiments). (I) Western blotting showing the expression of myelin proteins MBP and CNPase ( $n=3$ experiments). (J and $\mathbf{K}$ ) Quantification of the results in I. Data presented as mean \pm SD. ${ }^{*} P<0.05$, ${ }^{* *} P<0.01 \mathrm{vs}$. controls by paired $t$ test (B) or 1-way ANOVA with Tukey's post hoc test (D, F, H, I, and $\mathbf{K})$. Scale bars: $20 \mu \mathrm{m}$.

To explore whether Shc/MEK/ERK signaling is involved in the protective effects of LDLR in ischemic demyelination, U0126, a highly selective MEK inhibitor, was introduced into the culture medium of oligodendrocytes before the addition of differentiation medium. We found that U0126 compromised $\mathrm{MBP}^{+}$cells that were increased by $L d l r-G F P$ reporter virus infection (Figure 8, D and E; $P<0.001$ ). Immunoblotting further confirmed that NPVY-induced increases in MBP and CNPase expression coincided with the activation of Shc/MEK/ ERK signaling (Supplemental Figure 6). Nonetheless, U0126 restrained the expression levels of MBP, CNPase, p-MEK1/2, and p-ERK1/2 (Supplemental Figure 6). In addition, LDLR- 
A
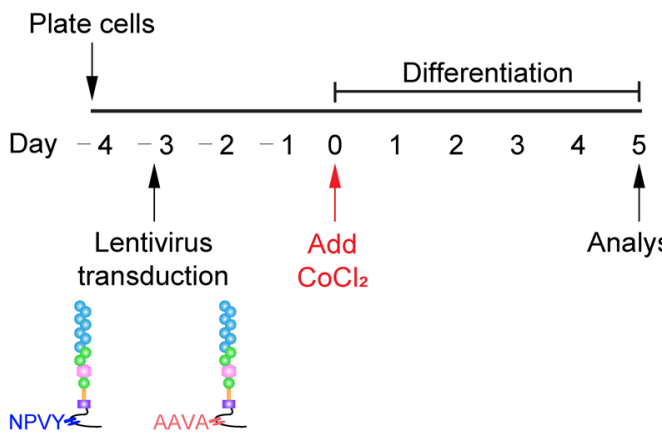

LDLR-wt LDLR-mut

B

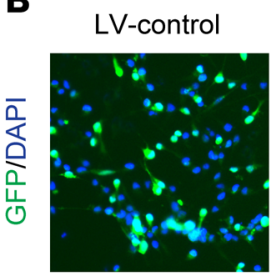

C

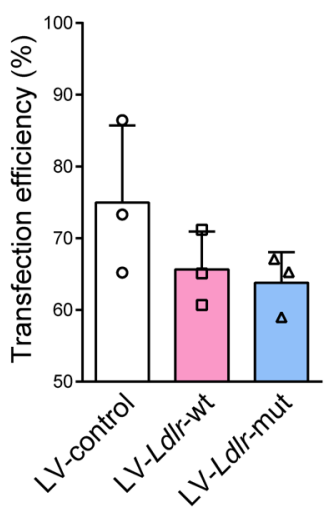

H

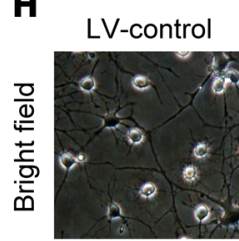

LV-Ldlr-wt

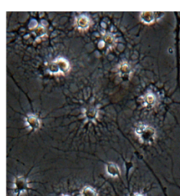

LV-Ldlr-mut
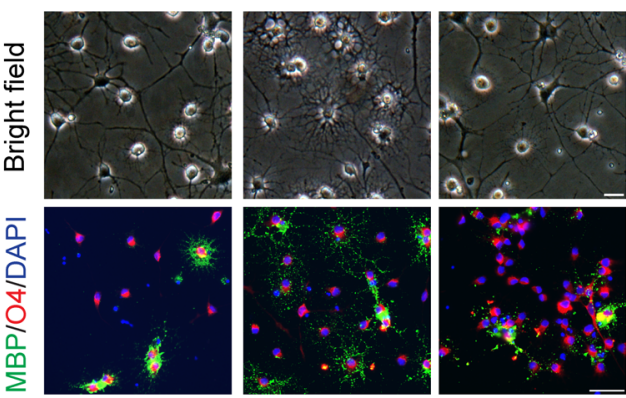

I
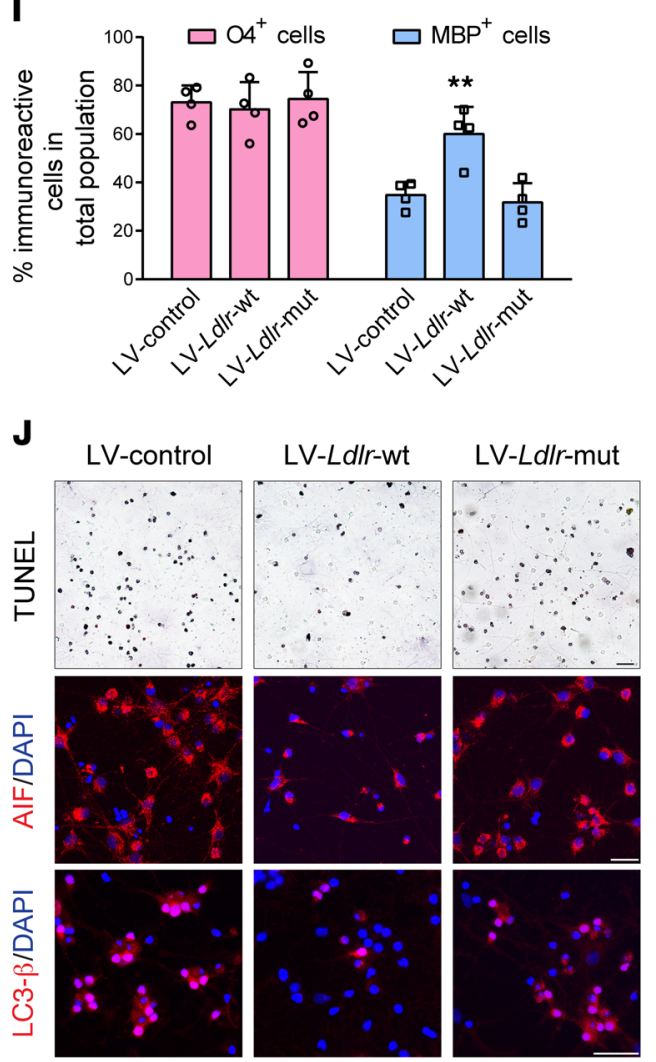

E

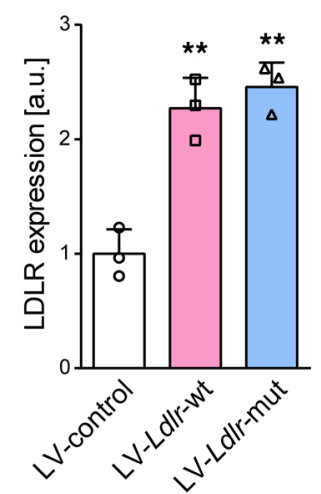

D

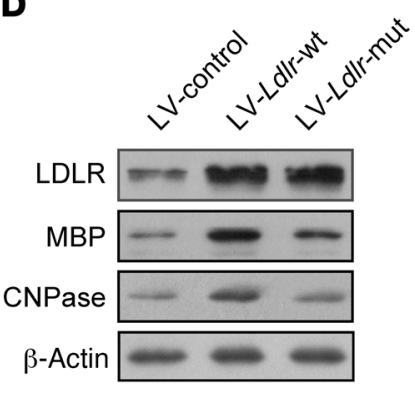

$\mathbf{J}$

LV-Ldlr-wt
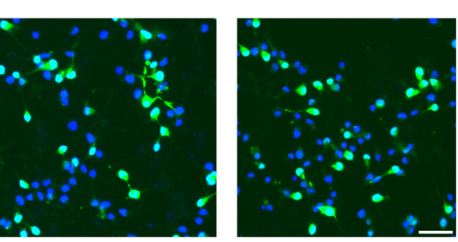

$\mathbf{F}$

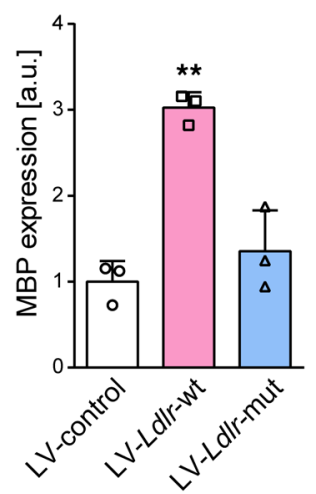

G

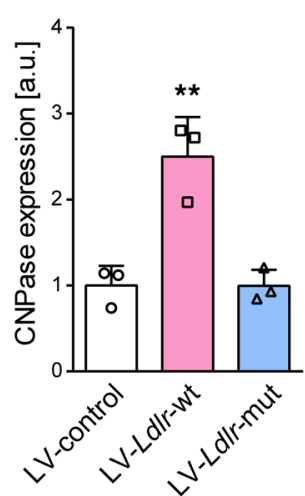

$\mathbf{K}$

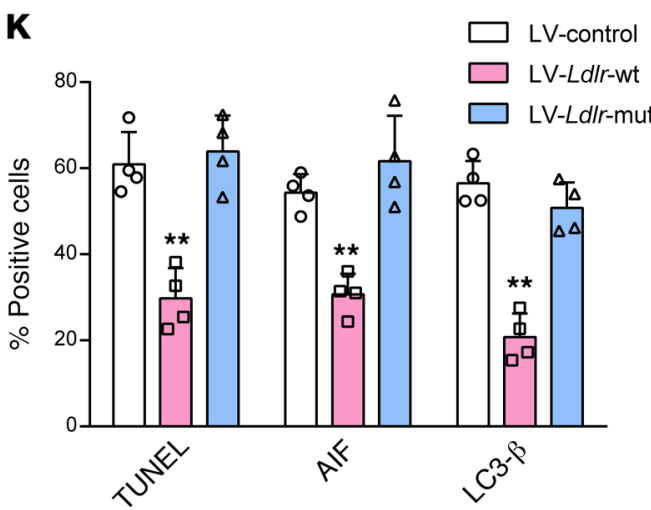

Figure 6. The NPVY motif is necessary in LDLR-induced attenuation of oligodendroglial disruption. (A) Experimental flow chart. (B and C) Representative fluorescence images and transfection efficiency analysis of OPCs transfected with GFP reporter lentiviruses (LV) ( $n=3$ experiments). (D) Western blotting showing the levels of LDLR and myelin proteins ( $n=3$ experiments). (E-C) Quantification of the results in $\mathbf{D}$. (H and I) Images and statistical analysis of the production of MBP+ oligodendrocytes ( $n=4$ experiments). ( $\mathbf{J}$ and $\mathbf{K}$ ) Images and statistical analysis of the survival of oligodendrocytes ( $n=4$ experiments). Data presented as mean $\pm S D .{ }^{* *} P<0.01$ vs. lentiviral control by 1-way ANOVA with Tukey's post hoc test. Scale bars: $20 \mu \mathrm{m}$. 
A

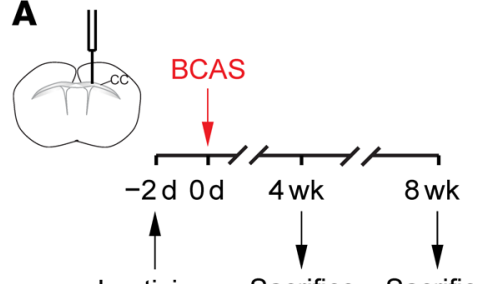
injection

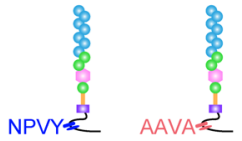

LDLR-wt LDLR-mut
Lentivirus Sacrifice Sacrifice

B
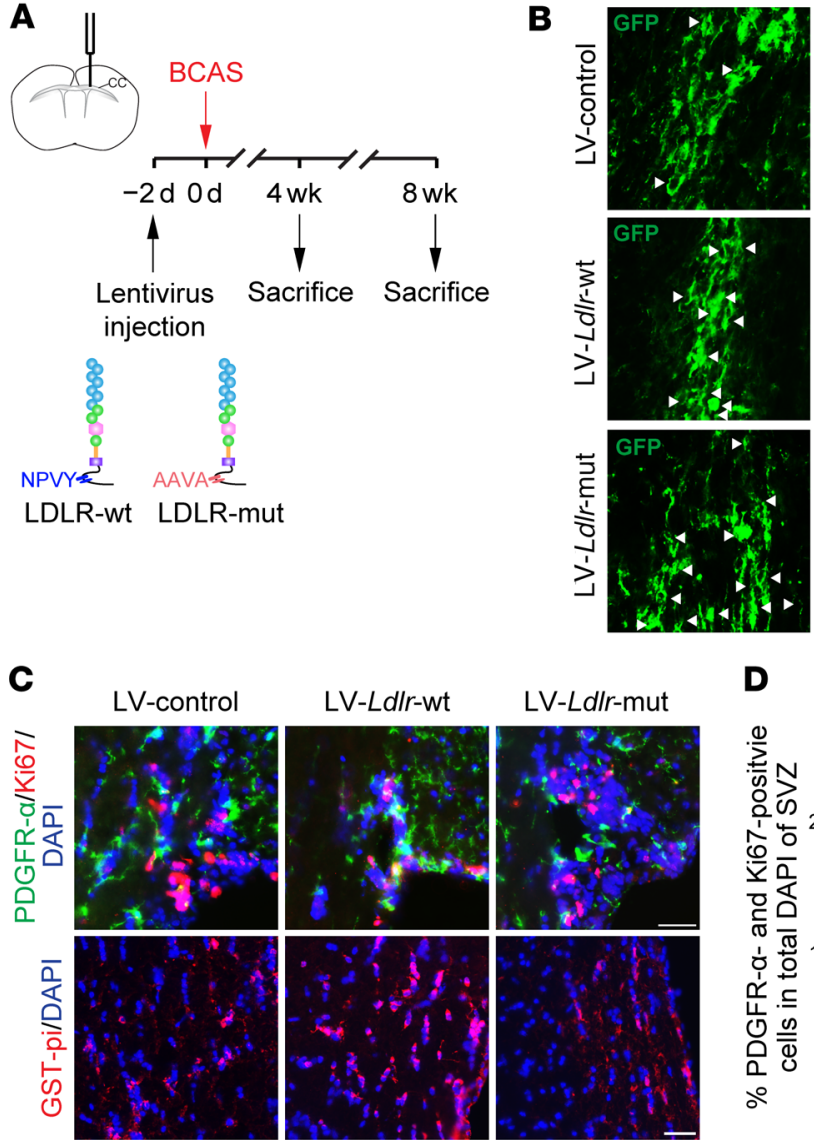

LV-Ldir-wt

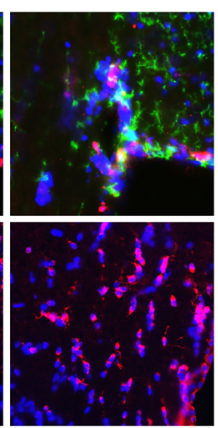

LV-Ldlr-mut

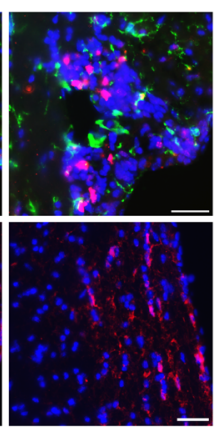

D
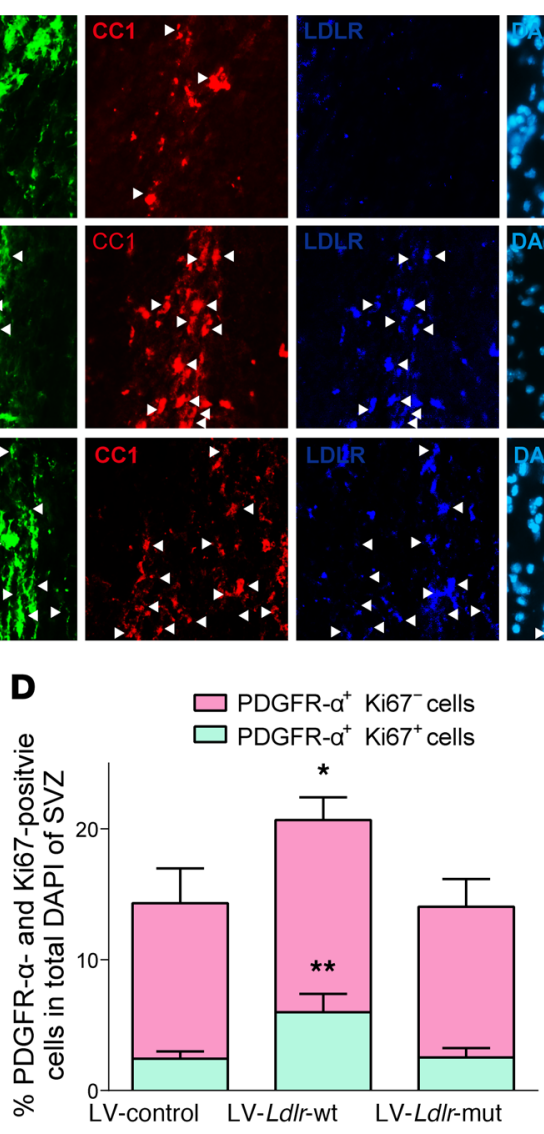

4445 atsint

E

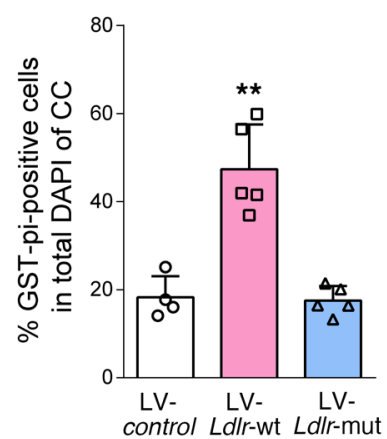

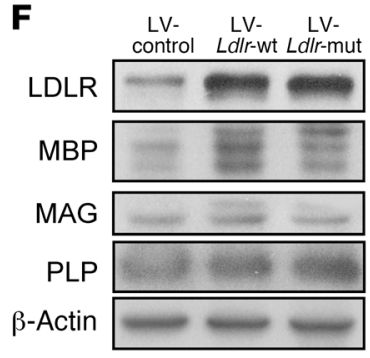

I

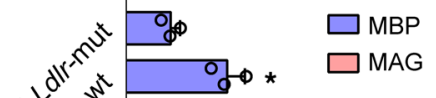

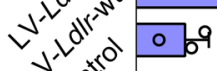

vis

divin

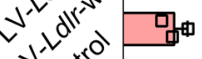

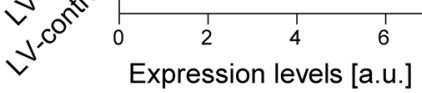

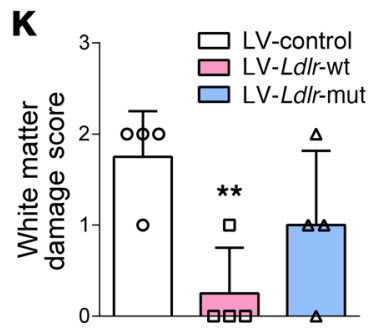

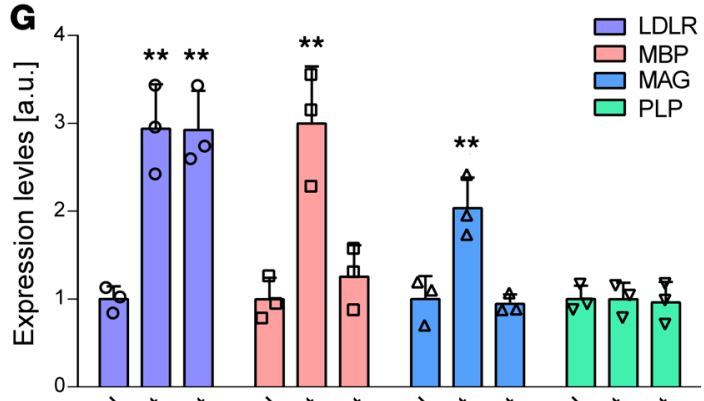

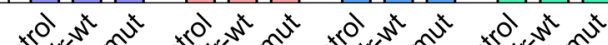

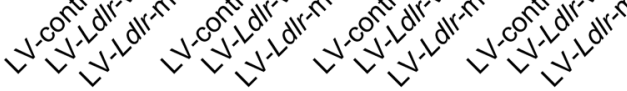

J

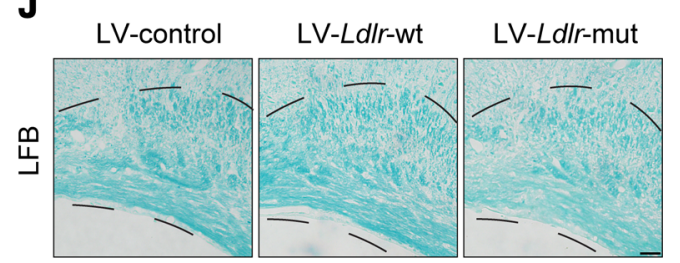

$\mathbf{L}$

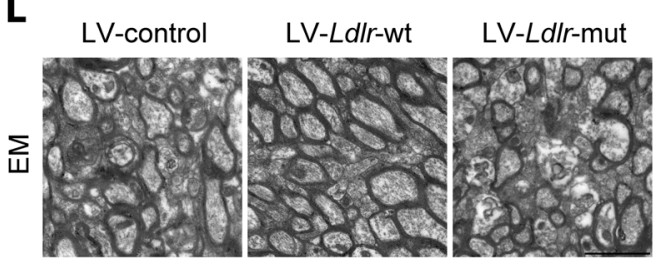

H

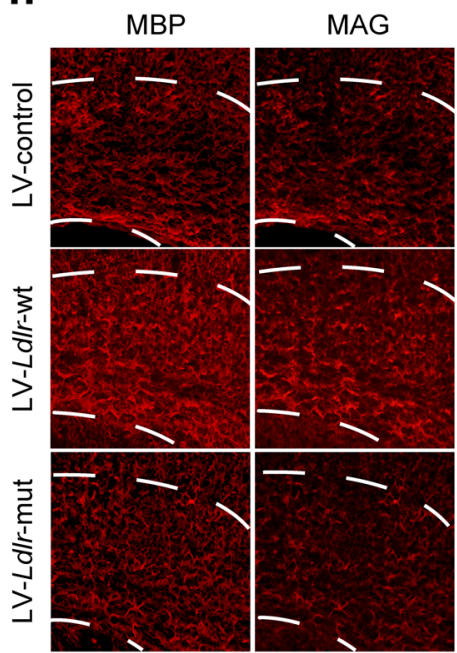

M

$\square$ LV-control $\square$ LV-Ldlr-wt $\square$ LV-Ldlr-mut

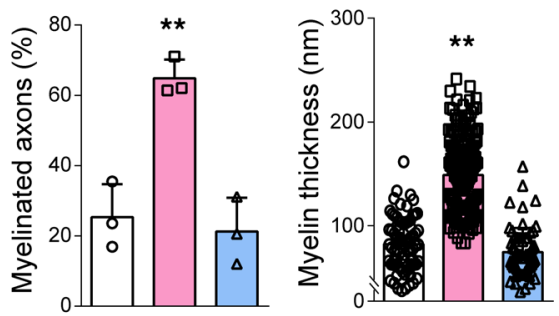


Figure 7. The NPVY motif is necessary in LDLR-induced alleviation of demyelinating injuries in the CC. (A) Experimental flow chart. (B) Representative fluorescence images of the transfection of GFP (green) reporter lentiviruses (LV) in mature oligodendrocytes (CC1+, red) with increased LDLR (dark blue) (indicated by white arrows) expression in the CC of BCAS mice. (C) In vivo OPC (PDGFR- $\alpha^{+}$, green) proliferation (Ki67+, red) and mature oligodendrocytes (CST- $\mathrm{pi}^{+}$, red) in mice receiving lentiviruses carrying Ldlr compared with control lentivirus ( $n \geq 3$ experiments). ( $\mathbf{D}$ and E) Quantification of the results in C. SVZ, subventricular zone. (F and $\mathbf{G}$ ) Immunoblotting analysis and its quantification demonstrating LDLR and myelin protein expression ( $n=3$ in each group). (H-M) Representative MBP, MAG, Luxol fast blue (LFB) staining, EM images, and quantification in the CC of BCAS mice receiving lentivirus encoding null, WT, and mutated Ldlr ( $n \geq 3$ in each group). Data presented as mean \pm SD. ${ }^{*} P<0.05,{ }^{* *} P<$ 0.01 vs. LV-control by 1-way ANOVA with Tukey's post hoc test. Scale bars: $20 \mu \mathrm{m}(\mathbf{B}, \mathbf{C}, \mathbf{H}$, and $\mathbf{J})$ and $2 \mu \mathrm{m}(\mathbf{I})$.

mediated oligodendrocyte survival was also abolished by U0126 treatment (Figure 8, F-H).

Ablation of LDLR impairs myelination in vivo. Ldlr-1- mice showed a 2.2-fold higher level of plasma cholesterol than WT counterparts (Figure 9A; $t=6.645, P=0.007$ ). However, the cholesterol concentrations in the CC were not significantly affected upon $L d l r$ ablation (Figure 9B; $t=0.298, P=0.785$ ). Specifically, $\mathrm{Ldll}^{-1-}$ mice had fewer proliferating OPCs and mature oligodendrocytes (Figure 9, C-E). Fiber tractography revealed intact fiber trajectories in various directions in the CC of WT mice; however, genetic ablation of $L d l r$ impaired fiber tract connectivity (Figure 9F), reduced fiber tracts (Figure 9G; $t=4.075, P=0.015$ ), and led to a decreased fractional anisotropy (FA) value (Figure 9, $\mathrm{H}$ and I; $t=4.988, P=0.008$ ), which was sensitive to changes in axonal integrity. In $L d l r$-deficient mice, myelin protein levels and positive signals and neurofilaments were remarkably decreased to less than half of normal levels (Supplemental Figure 7, A-D). White matter damage score was 1.5-fold higher in the CC (Supplemental Figure 7, E and F; $t=-6.708, P=0.001$ ). Additionally, fewer myelinated axons with thinner myelin sheaths were produced under the LDLR-deficient conditions (Supplemental Figure 7, G-I). It is noteworthy that the phosphorylation of Shc, MEK1/2, and ERK1/2 was downregulated in $L d l r^{-/}$mice (Supplemental Figure 7, J and K).

Genetic ablation of Ldlr in OPCs provokes aberrant differentiation and myelination. To focus on the oligodendroglial LDLR function, we then sought to examine the ability of $L d l r^{--}$OPCs to differentiate and to myelinate axons. In cultured $\mathrm{Ldlr}^{-1}$ OPCs, differentiation was remarkably suppressed, as indicated by lower levels of MBP and CNPase (Figure 10, A and B), immature light microscopic appearance (Figure 10C), and reduced $\mathrm{MBP}^{+}$cells (Figure 10D). We cocultured $\mathrm{Ldlr}^{-1-}$ OPCs with WT neurons. Upon differentiation, $\mathrm{Ldlr}^{--}$oligodendrocytes failed to produce discernable myelin wrapping around neurites, which was in contrast with abundant myelin formed in the WT coculture system (Figure 10E). Ldl//- oligodendrocytes with neuron cocultures showed a significant decrease in the absolute amount of myelination compared with WT oligodendrocytes (Figure 10F; $t=4.150, P=0.001$ ).

We transferred WT or $L d l r^{-1}$ OPCs into WT mice subjected to BCAS to compare their myelination competence. Transplantation was performed 1 week after BCAS surgery (Supplemental Figure 8A). Transplanted prelabeled OPCs survived and redistributed over long distances of the CC (Supplemental Figure 8B). Ki67positive cells were detected in the transplanted $\mathrm{GFP}^{+}$area (Supplemental Figure 8C). No significant differences in the positivity rate were observed between WT and KO OPCs (Supplemental Figure $8 \mathrm{D})$. Animals with WT transplants exhibited more $\mathrm{CC}^{+}$oligodendrocytes in regions adjacent to the implantation site (Supplemental Figure 8, E and F). Of note, WT OPCs generated more GST-pi ${ }^{+}$and $\mathrm{MBP}^{+}$cells than $\mathrm{Ldll}^{-}{ }^{-}$OPCs (Figure 11, A-F; GST-pi ${ }^{+} \mathrm{GFP}^{+}$mean decrease, $18.4 \%$ vs. $5.2 \%, P=0.009 ; \mathrm{MBP}^{+} \mathrm{GFP}^{+}$mean decrease, $38.3 \%$ vs. $20.9 \%, P=0.029$ ). As a consequence, BCAS-induced demyelination was attenuated in the mice receiving WT OPC transplantation, but not $\mathrm{Ldlr}^{-}$OPCs (Figure 11, G-I). These results indicated an indispensable role of oligodendroglial LDLR in myelin restoration against chronic cerebral ischemia.

Supplementation of LDLR with NPVY motif rescues myelination in the LDLR-deficient condition. We then asked whether supplementation of LDLR favored the restoration of myelination in the $\mathrm{Ldlr}^{-1}$ system, and if so, whether this protection was dependent on a functional NPVY domain. Cultured $L d l r^{-}$OPCs could regain normal differentiation under transfection with lentivirus expressing WT NPVY. Ldlr ${ }^{--}$OPCs transfected with WT Ldlr expressing the NPVY motif yielded greater MBP and CNPase expression than those re-expressing $L d l r$ with the mutated AAVA motif (Supplemental Figure 9, A-D). This improvement was accompanied by greater levels of p-Shc, p-MEK1/2, and p-ERK1/2 (Supplemental Figure 9, C and E). As for $L d l r^{--}$mice, we evaluated the effects of LDLR supplementation into the CC 2 weeks before euthanasia (Supplemental Figure 10A). Specifically, both WT and mutant lentiviruses could transfect mature oligodendrocytes and restore their expression of LDLR (Figure 12A). LDLR with the NPVY motif facilitated local OPC proliferation and increased the number of mature oligodendrocytes (Figure 12, B-E). Consequently, a white matter network of increased fiber connectivity with more fiber tracts was achieved after WT $L d l r$-GFP virus treatment (Figure 12, F-H). Immunostaining and immunoblotting of myelin-associated proteins revealed that WT LDLR supplementation was able to mitigate myelin loss in $\mathrm{Ldlr}^{-}$mice (Figure 12, I-K, and Supplemental Figure 10, B and C). In addition, WT LDLR induced Shc/MEK/ ERK pathway initiation in the CC, as manifested by remarkably elevated levels of p-Shc, p-MEK1/2, and p-ERK1/2 (Supplemental Figure 10, B and D). Myelinated axons and myelin sheath thickness were significantly retained by LDLR with the NPVY motif (Supplemental Figure 10, E and F). However, mutated LDLR with the AAVA motif did not exert any detectable improvement in the restoration of myelination.

\section{Discussion}

LDLR has a long-established critical role in cholesterol metabolism. In this study, we identified a functional activity of LDLR in the pathogenesis of ischemic demyelination, which may involve the intracellular NPVY motif and subsequent Shc/MEK/ ERK pathway. Hypoxic-ischemic injury caused oligodendroglial LDLR aberrance and impaired oligodendroglial differentiation, survival, and subsequent myelination. Most importantly, the restoration of myelination we noted in the study, through increased 
A

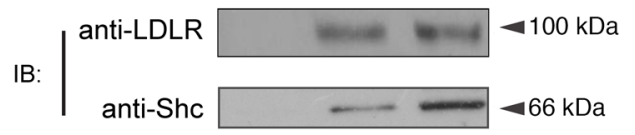

IB: $\begin{aligned} & \text { anti-LDLR } \square-100 \mathrm{kDa} a \\ & \text { anti-Shc } \square+\longrightarrow 66 \mathrm{kDa}\end{aligned}$

C

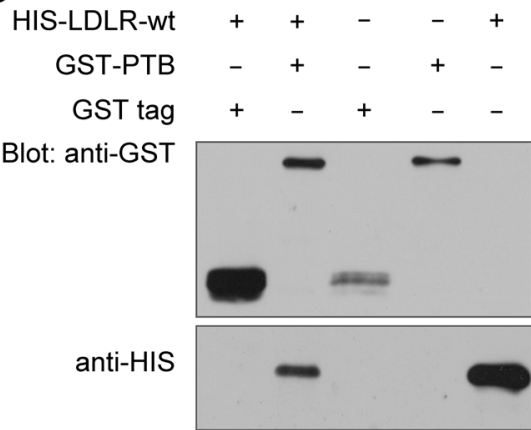

D
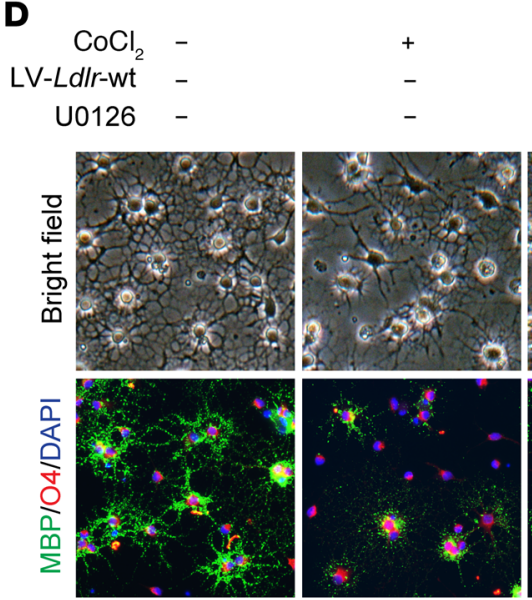

$\mathbf{F}$

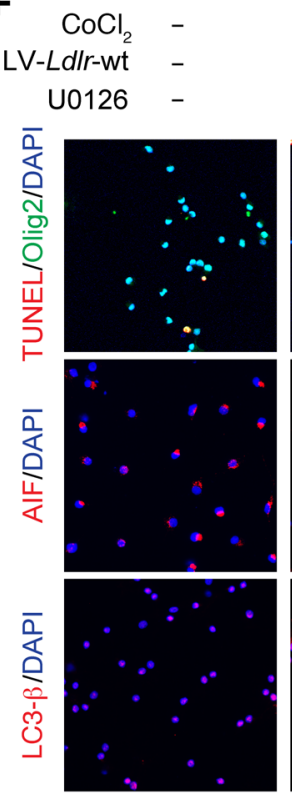

B
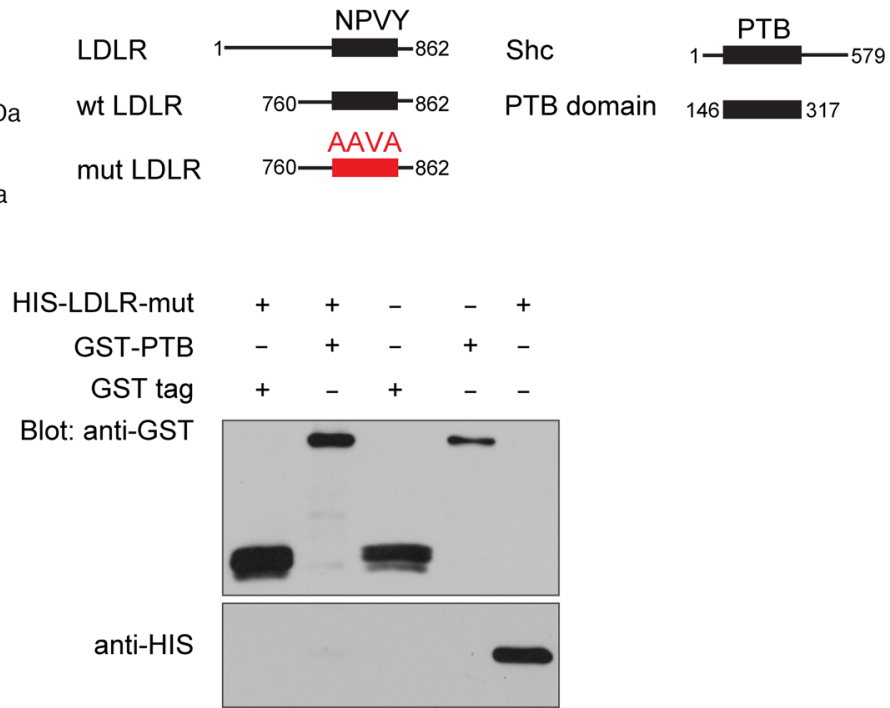

E
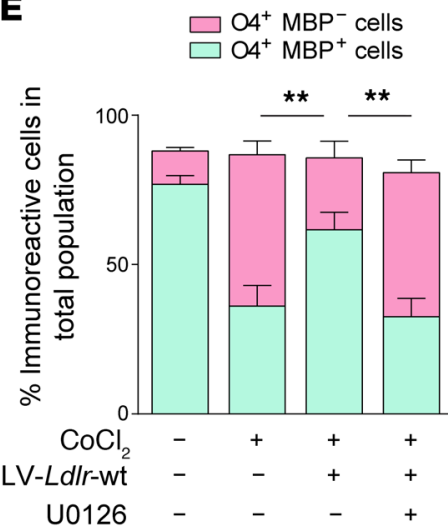

य. 8

G

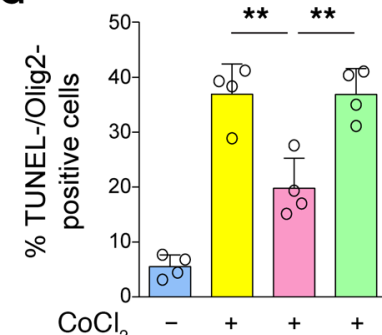

LV-Ldlr-wt - - + + U0126 - - - +

H

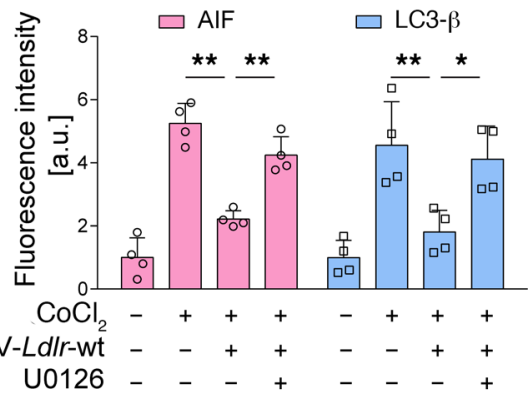


Figure 8. LDLR protects against demyelination by binding Shc and activating downstream MEK/ERK signaling. (A) Immunoassay of lysates of cerebral CC tissue after immunoprecipitation with LDLR, analyzed by immunoblotting (IB) with anti-LDLR and anti-Shc ( $n=3$ in each group). (B) Schematic diagrams of LDLR, Shc, and constructed LDLR and Shc fragments. (C) GST pull-down assay revealing the interaction between LDLR and Shc ( $n=3$ in each group). ( $\mathbf{D}$ and $\mathbf{E})$ Representative images of light microscopy and MBP/O4 staining ( $n=4$ experiments). (F-H) Representative images and the quantification of cell death signal staining, including TUNEL, AIF, and LC3- $\beta$ ( $n=4$ experiments). Data presented as mean $\pm \mathrm{SD}$. ${ }^{*} P<0.05$, ${ }^{*} P<0.01$ by 1 -way ANOVA with Tukey's post hoc test. Scale bars: $20 \mu \mathrm{m}$.

expression of LDLR with its NPVY motif, suggests an important function of LDLR in adulthood myelination. Oligodendrocyte-originated miR-344e/410-3p may act as the upstream regulator of LDLR. Human miR-410-3p, sharing a common preserved seed region sequence with mouse miR-344e-3p, was shown to be remarkably increased in patients with leukoaraiosis, supporting the translation of these findings in rodents to human biology.

We used BCAS surgery with microcoils to induce ischemic demyelination and prolonged it to 8 weeks to capture a bigger picture of the pathological process. Consistent with the previous study $(17,18)$, a pronounced lack of axonal myelination was noted with axonal loss in the CC. In the adult central nervous system (CNS), OPCs are abundant and always ready to proliferate to replace oligodendrocytes and myelin (19). At 4 weeks after BCAS, OPCs were found to mobilize, with increased proliferative abil- ity. However, under hypoxia, OPC proliferation was gradually arrested and remained so until the 8-week endpoint. Thus, with the effects on OPC mobilization and substantial oligodendrocyte death, BCAS-induced demyelination was constantly prolonged and augmented.

$L d l r$ is expressed in all types of neural cells based on the brain-RNA-seq database (20). We found that LDLR was highly expressed in OPCs and mature oligodendrocytes as compared with other cultured neural cells, including astrocytes, neurons, and microglia. Oligodendroglial cells express LDLRs to endocytose sterols packaged in lipoprotein particles, probably for utilization in adulthood myelination $(21,22)$, establishing a clear role for LDLR in myelin biosynthesis in the CNS. Specifically, we compared the ability of WT and $\mathrm{Ldlr}^{-/}$oligodendrocytes to myelinate axons. WT neurons cocultured with $\mathrm{Ldlr}^{--}$OPCs displayed decreased myelinated axon lengths compared with WT OPCs. In addition, transplantation of $\mathrm{Ldlr}^{-/}$OPCs did not ameliorate BCAS-induced hypomyelination in WT mice. These data suggested that the function of LDLR in oligodendrocytes is cell autonomous. This conclusion is also supported by the observation that lentiviral vector-induced upregulation or reexpression of LDLR in oligodendrocytes resulted in the restoration of myelination in the CC.

According to previous reports (23-27) and our findings, although genetic ablation of $L d l r$ led to a moderate (2.2-fold) elevation in plasma cholesterol levels, brain cholesterol concentrations remained unaltered in $\mathrm{Ldlr}^{-/}$mice. Because of the very restricted
A

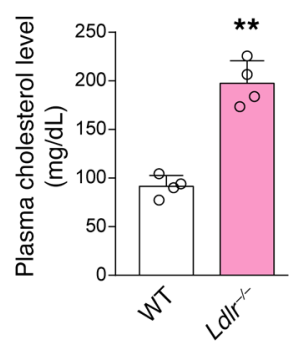

B

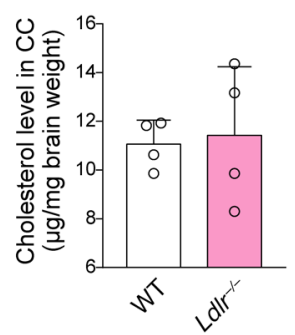

F

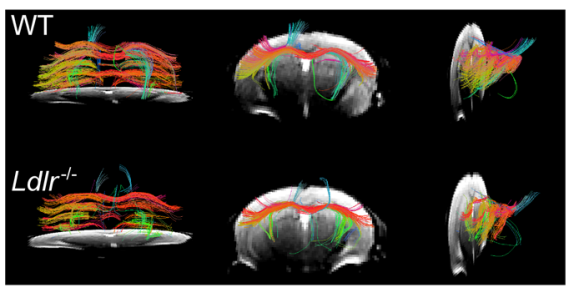

C

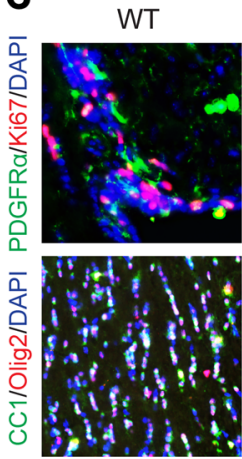

G

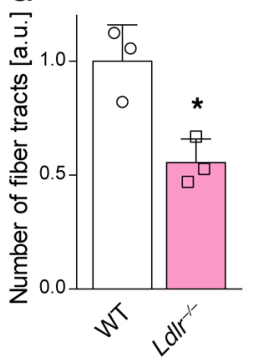

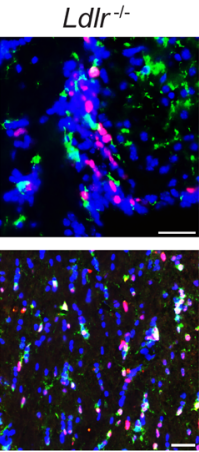

H

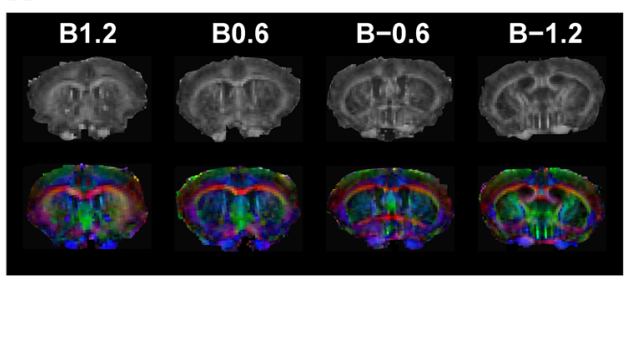

D

E

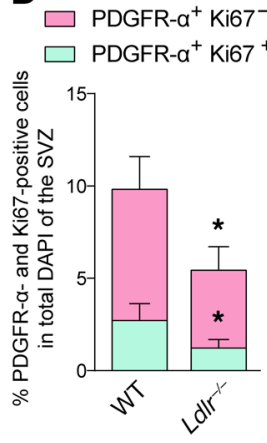

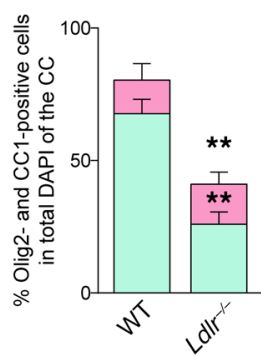

I

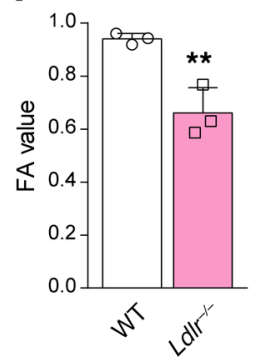

Figure 9. Absence of LDLR results in aberrant myelination in vivo. (A and B) Plasma and local CC cholesterol contents of WT and $L d l r^{-1-}$ mice ( $n=4$ in each group). (C) Immunofluorescence images of proliferated OPCs (PDCFR- $\alpha^{+} \mathrm{Ki}^{+} 7^{+}$cells) in the subventricular zone and mature oligodendrocytes (Olig2 ${ }^{+}$CC $1^{+}$cells) in the CC ( $n=4$ experiments). ( $\mathbf{D}$ and $\left.\mathbf{E}\right)$ Quantification of the results in C. (F) Representative fiber tractographies obtained from the CC of WT and $L d l r^{-1-}$ mice ( $n=3$ in each group). (G) Quantification of the results in $\mathbf{D}$ and $\mathbf{E}$. (H) Colored principal eigenvector maps of mouse brain and (I) FA value in Ldlr $r^{-1-}$ mice compared with WT mice ( $n=3$ in each group). The numbers (B1.2, B0.6, B-0.6, B-1.2) refer to the distance (mm) from the bregma, with plus and minus being either anterior or posterior to the bregma. Eigenvector components were mapped to the following color schemes: red = left-right, blue = cranial-caudal, and green $=$ dorsal-ventral directions. Data presented as mean \pm SD. ${ }^{*} P<0.05,{ }^{*} P<0.01$ vs. WT mice by paired $t$ test. Scale bars: $20 \mu$ m. 
A

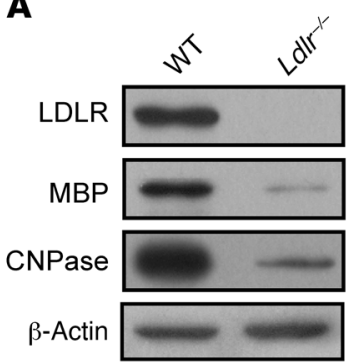

B

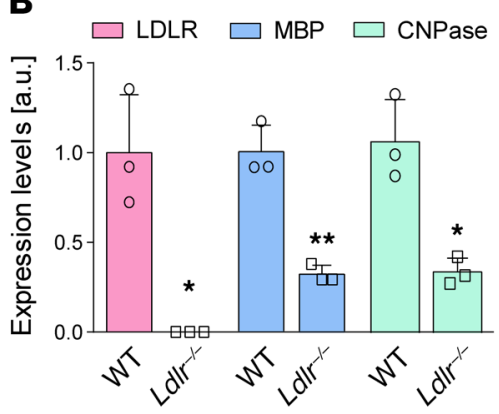

C

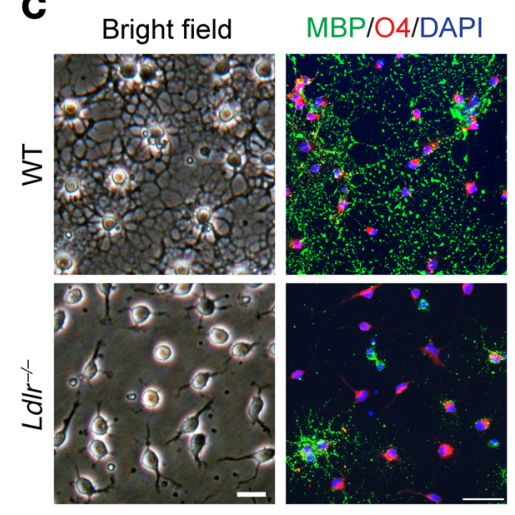

D

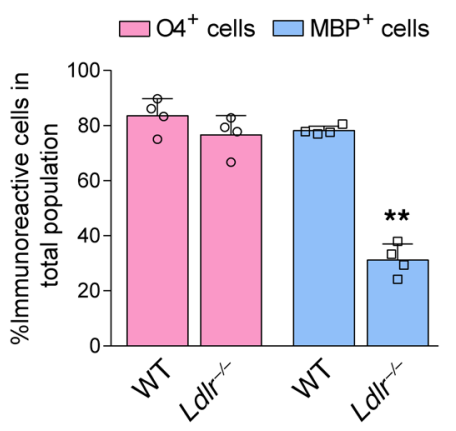

E

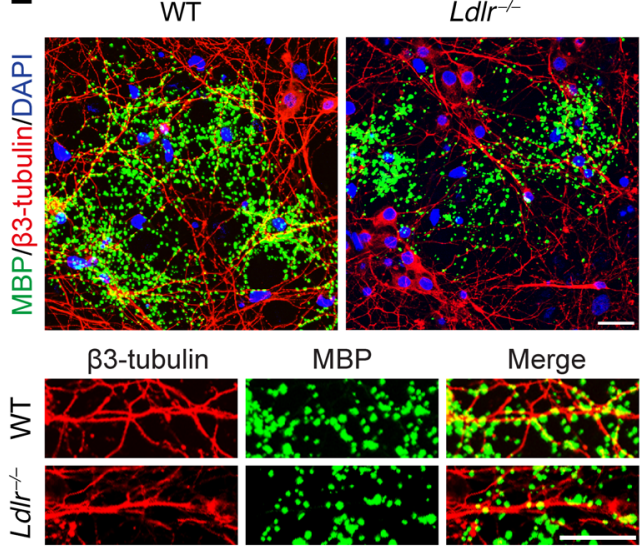

$\mathbf{F}$

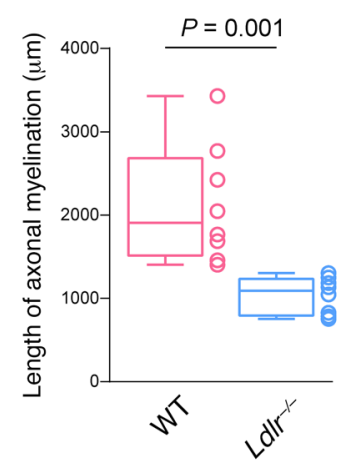

Figure 10. Absence of LDLR results in aberrant myelination in vitro. (A) Immunoblotting for LDLR, MBP, and CNPase expression in oligodendrocytes of 2 genotypes ( $n=3$ experiments). (B) Quantification of the results in A. (C) Representative light microscopic images of MBP/O4 staining of WT and $L d l r^{-1-}$ oligodendrocytes ( $n=4$ experiments). (D) Quantification of the results in C. (E) Representative MBP and $\beta 3$-tubulin staining images of the coculture system composed of WT neurons with WT or Ldlr $r^{-1-}$ oligodendrocytes ( $n=8$ experiments). (F) Quantification of the results in $\mathbf{E}$. Data presented as mean \pm SD. ${ }^{*} P<0.05,{ }^{* *} P<0.01$ vs. WT oligodendrocytes by unpaired $t$ test. Scale bars: $20 \mu \mathrm{m}$

entry of systemic sterols into the CNS, locally synthesized cholesterol becomes the main factor affecting myelination (7). However, the synthesis rate of brain cholesterol in $\mathrm{Ldlr}^{-/}$mice was reported to be unchanged as well (23). Therefore, using $\mathrm{Ldlr}^{-1-}$ mice in the present study may have excluded the possible confounding effects exerted by either systemic or local cholesterol. As for the effects of LDLR on cholesterol levels in oligodendrocytes, we found that hypoxia-induced LDLR reduction did not lead to alterations in tissue and cell cholesterol content. Oligodendroglial ability to take up LDL was also not affected. This could be explained by a reduction in the ATP binding cassette transporter A1 (ABCA1), which mediates cholesterol efflux in cells (28). Lack of the LDLR has been reported to impair ABCA1 expression by a sterol regulatory element-binding protein 1-dependent mechanism (29). We can also attribute this observation to either an increased local cholesterol synthesis in oligodendrocytes or to a compensatory elevated level of other candidates, such as LRPs and caveolae, for LDL uptake. Taken together, rather than focusing on cholesterol regulation, these observations prompted us to address the possibility of other potentially functional domains in LDLR that may be engaged in ischemia-induced demyelination.

LDLR is the prototype of the LDLR family. Except for the transmembrane anchor and motifs responsible for binding or releasing ligands, LDLR contains an NPVY motif in its cytoplasmic domain (14). This motif is conserved in the LDLR of species ranging from frogs to humans (30). The phosphorylation on the Tyr of the NPVY motif of LDLR provides a docking site for proteins containing a PTB domain, such as a putative protein of autosomal recessive hypercholesterolemia (ARH) (31). The NPVY motif has been widely recognized as requisite for endocytosis of LDLR by interaction with adaptor proteins (32), such as ARH (30) and sorting nexin 17 (SNX17) (33). We demonstrated that cytoplasmic Shc can interact with the NPVY motif through its PTB domain in oligodendrocytes. This interaction functioned as an obligate component in oligodendroglial differentiation, survival, and myelination. These data suggest that the NPVY motif within the tail of LDLR may function not only as an internalization signal but also as a binding motif for cell molecular components involved in signal transduction.

We demonstrated that the aberrant LDLR induced remarkable inhibition of the Shc/MEK/ERK pathway, which was responsible for oligodendroglial death following chronic cerebral ischemia. This observation is in accordance with the observation that the silencing of LDLR significantly suppressed ERK activation in tumor cells $(34,35)$. In addition, the functional integrity of LDLR is important for cell survival $(36,37)$, as the rescued ERK initi- 
A

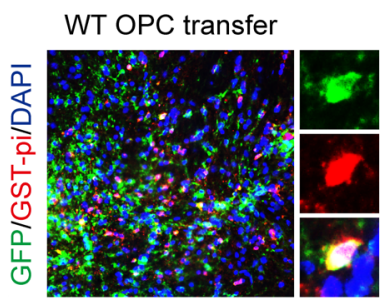

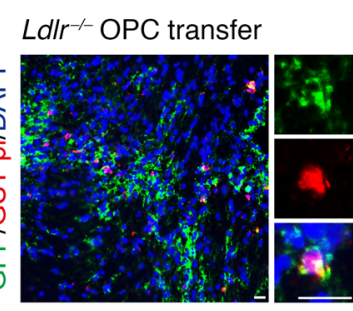

B

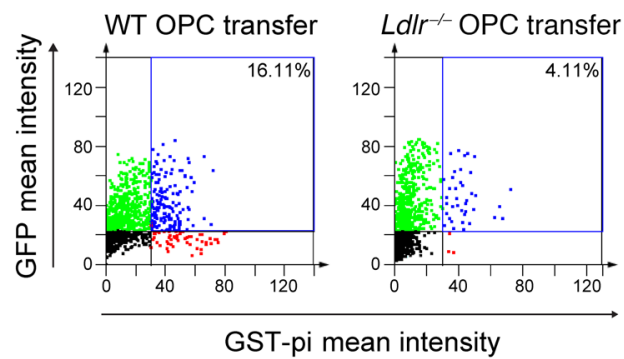

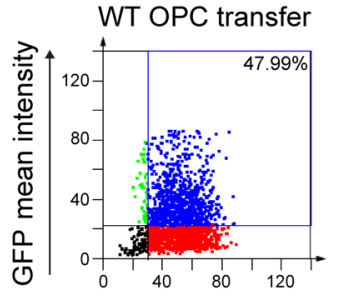

E
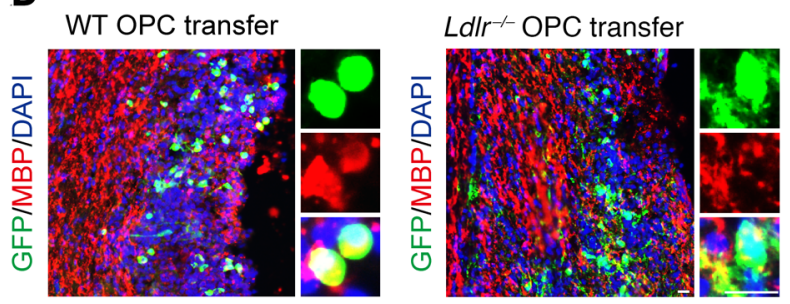

C
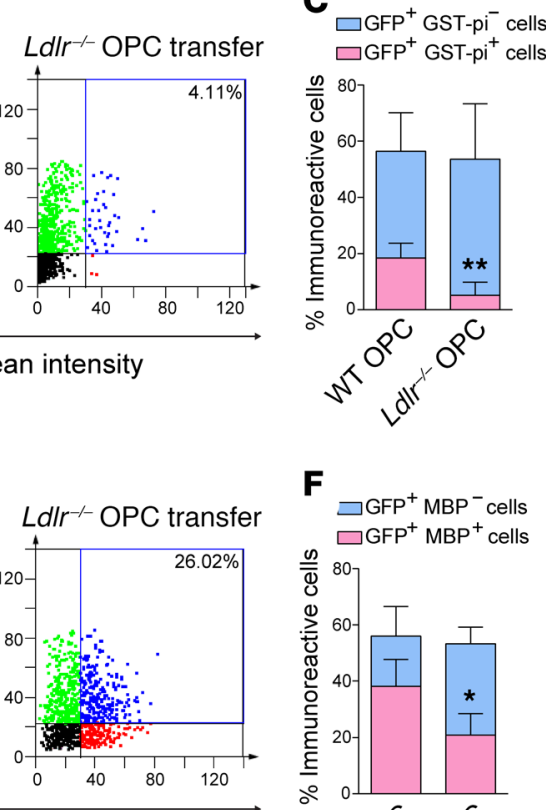

MBP mean intensity

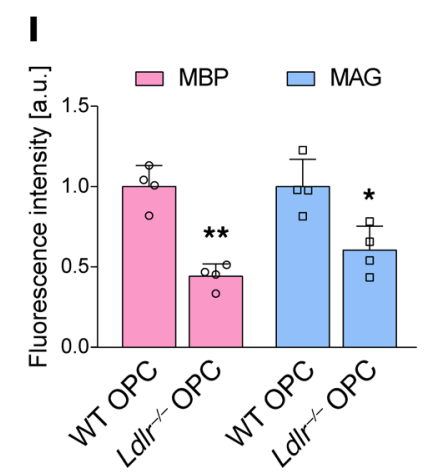

$\mathbf{F}$

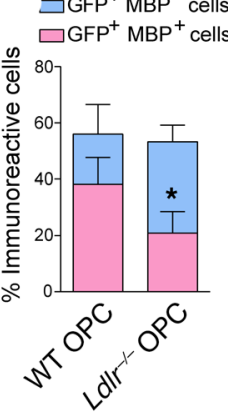

G

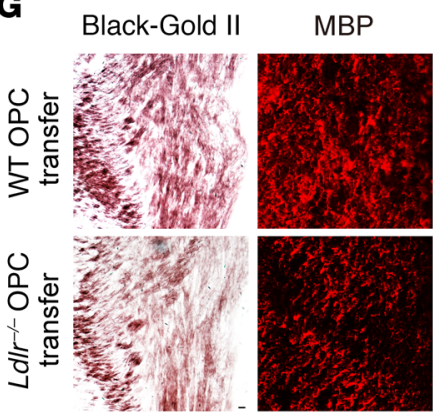

MAG

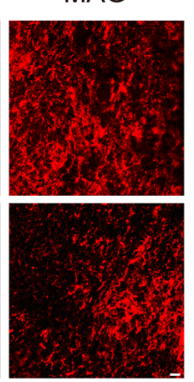

H

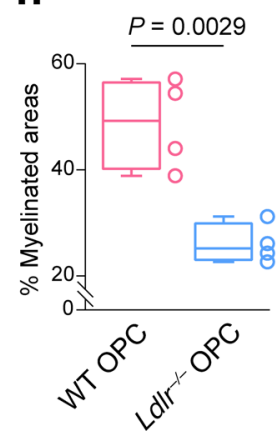

Figure 11. Transplantation of $L d I r^{\prime-}$ OPCs into the CC could not ameliorate demyelination in BCAS mice. (A and D) Representative images showing the differentiation ability of OPCs transplanted into the CC of BCAS mice ( $n=4$ in each group). (B and C) Quantification of the results in A. (E and F) Quantification of the results in D. (G) Representative Black-Gold II, MBP, and MAC staining of the CC in mice injected with WT or LdIr ${ }^{1-}$ OPCs ( $n=4$ in each group). (H and I) Quantification of the results in G. Data presented as mean \pm SD. ${ }^{*} P<0.05,{ }^{* *} P<0.01 \mathrm{vs}$. WT OPCs by unpaired $t$ test. Scale bars: $10 \mu \mathrm{m}$.

ation cannot be obtained with mutated NPVY. The Shc/MEK/ ERK pathway is also known to control myelin synthesis in the CNS (38-40). LDLR deficiency diminished the activation of Shc/ MEK/ERK, and therefore impaired normal myelination both in vitro and in vivo. Supplementation of LDLR can reverse LDLR deficiency- or hypoxia-related demyelination by activation of Shc/MEK/ERK signaling, which can regulate the production of several myelin proteins, including MBP and MAG $(40,41)$, and increase myelin thickness (38).

In summary, this study identifies a functional activity of LDLR in the pathogenesis of ischemic demyelination. LDLR, negatively regulated by miR-344e/410-3p, binds to Shc via its NPVY motif, stimulates the Shc/MEK/ERK axis, and restores myelination. This NPVY-Shc interaction, which is disrupted by chronic ischemia, is necessary for oligodendroglial maturation and myelin formation. Our findings may provide a basis for modulating the miR-344e/410$3 \mathrm{p} / \mathrm{LDLR} / \mathrm{Shc}$ axis in the treatment of demyelinating diseases or other neurological disorders involving white matter pathologies.

\section{Methods}

Mice. Male adult C57BL/6 mice weighing 24-29 g, female mice at 16-18 days of pregnancy, and $\mathrm{Ldlr}^{-1-}$ mice $\left(L d l^{\text {mICd82 }} / \mathrm{Nju}, \mathrm{C} 57 \mathrm{BL} / 6\right.$ background; T001464) were purchased from the Model Animal Research Institute of Nanjing University.

Study population and data collection. From July 2018 to October 2018, we enrolled 187 subjects who were more than 45 years old and referred to the neurology inpatient department at Jinling Hospital for further assessment due to nonspecific headaches without migraines and dizziness. All subjects underwent a careful general physical and neurological examination as well as MRI test. No abnormal neurological signs were detected in the recruited participants. Baseline data including age, sex, and history of hypertension, diabetes, and hyperlipemia were recorded. The exclusion criteria were as follows: (a) history of stroke, brain trauma, intracranial tumor, CNS infection, active malignancy, thyroid diseases, autoimmune diseases, active or chronic inflammatory diseases, severe liver and renal function impairment; (b) signs of neurological deficit; and (c) contraindication for MRI examination. 
A
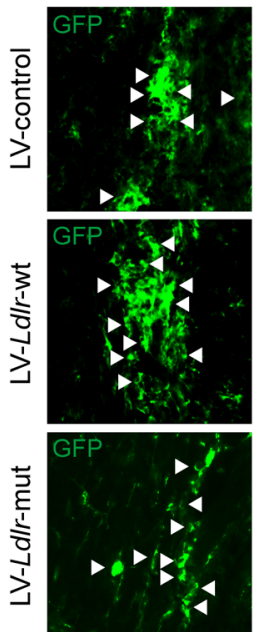

D

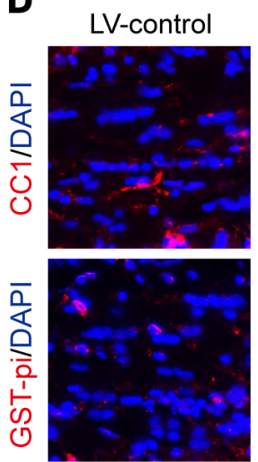

$\mathbf{F}$

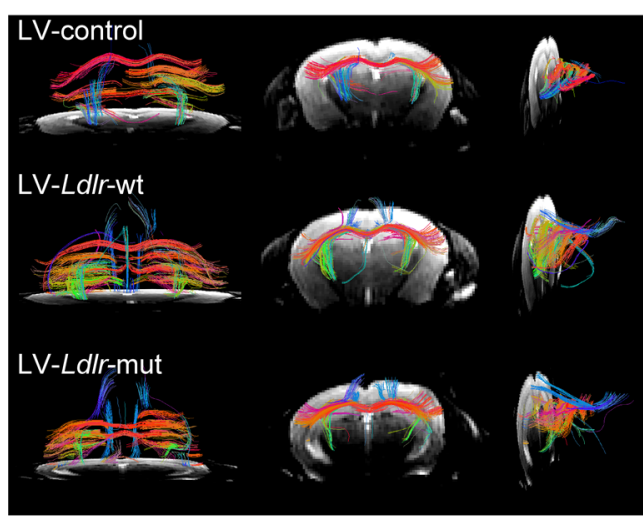

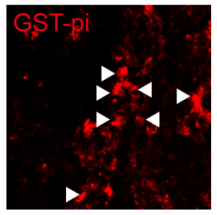
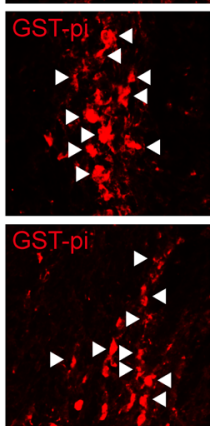

LV-Ldlr-wt

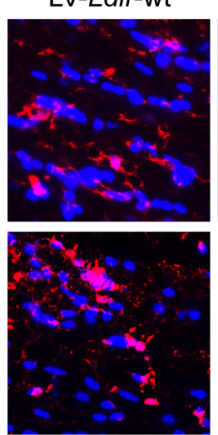

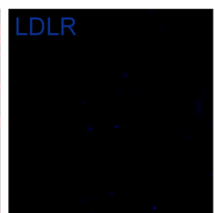
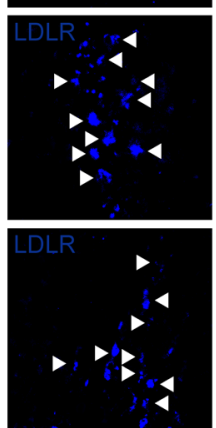

LV-Ldlr-mut

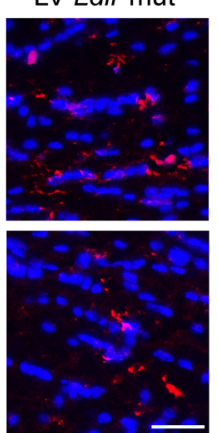

G

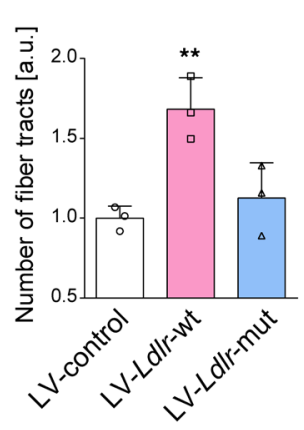

H
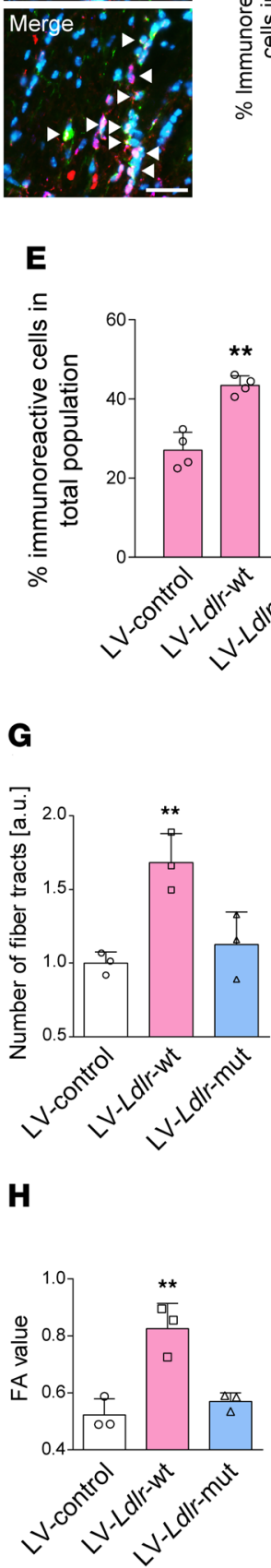

E
LV-control
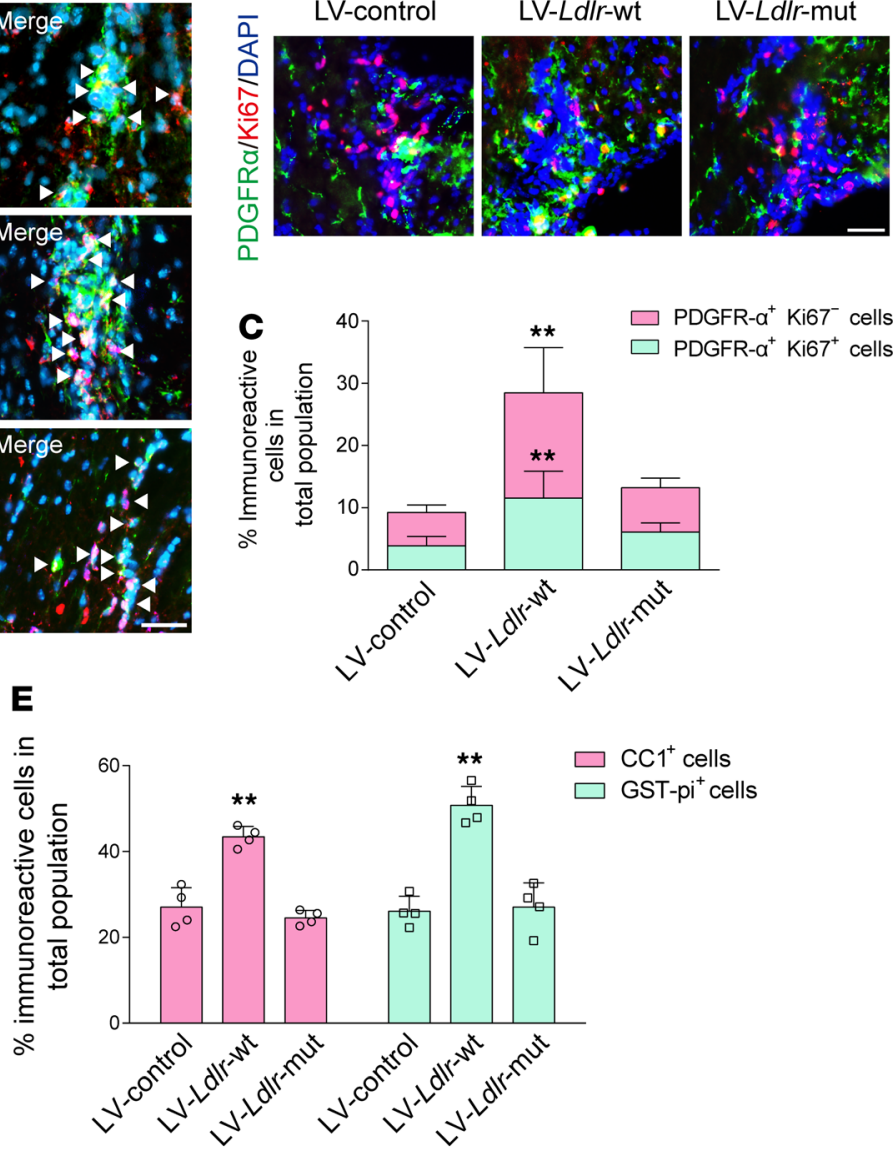

I

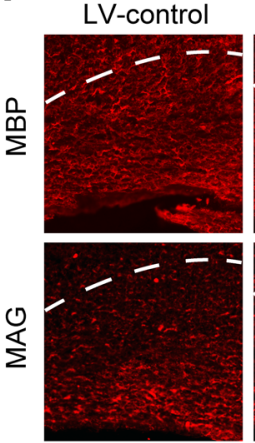

LV-Ldlr-wt

LV-Ldlr-mut
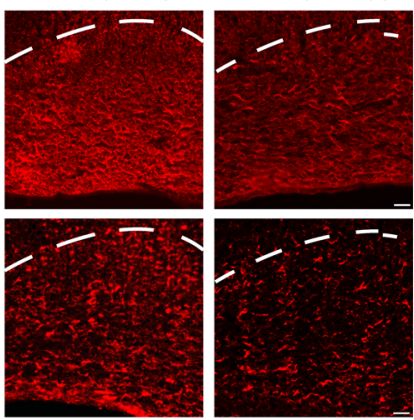

J

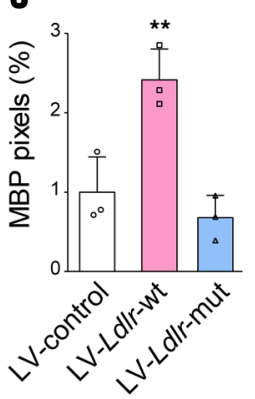

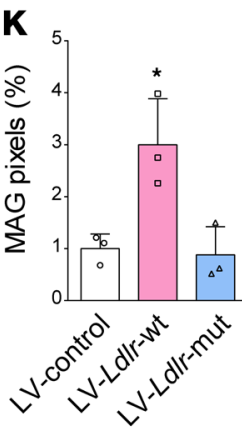


Figure 12. Reexpression of LDLR with NPVY motif rescues hypomyelination in $\mathbf{L d l l}^{-/-}$mice. (A) Representative fluorescence images of the transfection of GFP (green) reporter lentiviruses into mature oligodendrocytes (CST-pi ${ }^{+}$, red) with re-expression of LDLR (dark blue, indicated by white arrows) in the CC of $L d l r^{-1-}$ mice. (B) Images showing the proliferation of native OPCs ( $n=4$ in each group). (C) Quantification of the results in B. (D) Images showing the mature oligodendrocytes (CC1+ or CST-pi+ cells) in the CC ( $n=4$ in each group). (E) Quantification of the results in $\mathbf{D}$. (F) Representative fiber tractographies of mice injected with 3 types of viruses. Eigenvector components were mapped to the following color schemes: red = left-right, blue $=$ cranial-caudal, and green $=$ dorsal-ventral directions ( $n=3$ in each group). ( $\mathbf{G}$ and $\mathbf{H}$ ) Quantification of the results in F. (I) MBP and MAG staining of the CC in $\mathrm{Ldlr}^{-1-}$ mice receiving lentiviruses ( $n=4$ in each group). The CC boundary is indicated by the white dashed line. ( $\mathbf{J}$ and $\mathbf{K}$ ) Quantification of the results in I. Data presented as mean $\pm \mathrm{SD}$. ${ }^{*} P<0.05,{ }^{*} P<0.01$ vs. LV-control by 1 -way ANOVA with Tukey's post hoc test. Scale bars: $20 \mu \mathrm{m}$.

BCAS surgery. The BCAS model was established as previously described (42). Briefly, mice were deeply anesthetized with $2 \%$ isoflurane in 33\% oxygen. After exposing both CCs from their sheaths, a microcoil with $0.18 \mathrm{~mm}$ inner diameter (Sawane Spring Co.) was wound around the CC. Another microcoil of the same size was wound around the other CC 30 minutes later. The rectal temperature was maintained between $36.5^{\circ} \mathrm{C}$ and $37.5^{\circ} \mathrm{C}$ during surgery.

Cell culture and hypoxic treatment. Mixed glial cultures from 1- to 2-day-old WT or $\mathrm{Ldlr}^{-/-}$pups were grown for 7 days and the flasks were shaken for 1 hour on an orbital shaker $(200 \mathrm{rpm})$ at $37^{\circ} \mathrm{C}$ to remove microglia, followed by another 18- to 20-hour shake at $200 \mathrm{rpm}$. Adherent cells were collected to obtain astrocyte cultures. A high purity of OPCs was obtained as reported previously (43-45). In vitro hypoxia was induced by either chronic $\mathrm{CoCl}_{2}(10 \mu \mathrm{M}$ for 5 days) or OGD treatment ( 2 hours). $\mathrm{CoCl}_{2}$ was added with differentiation medium. As previously reported (46), after 2 hours of OGD, cells were returned to normal incubator conditions with differentiation medium. Microglia were shaken off and seeded onto plates after the mixed glial culture was maintained for 14 days. For MEK/ERK inhibition, OPCs were preincubated with $10 \mu \mathrm{M}$ U0126 (9903, Cell Signaling Technology) for 2 hours before the addition of differentiation medium. For myelination coculture (47), WT neurons were cultured on poly-D-lysine-coated coverslips for 4-5 days in maintenance medium. Separated WT or $\mathrm{Ldlr}^{-}$ OPCs were then added to the medium and the maintenance medium was changed to myelin coculture medium 24 hours later. Myelination was identified as regions of colocalization between MBP-positive oligodendrocyte processes and $\beta 3$-tubulin-positive neurites (48).

Virus production and stereotaxic injection. A cDNA encoding the WT Ldlr sequence and the EGFP reporter gene (pLV[Exp]-EGFP: T2A:Puro-EF1A>mLdlr [NM_010700.3]), and another encoding the mutated Ldlr sequence and EGFP reporter gene (pLV[Exp]-EGFP: T2A:Puro-EF1A>[mLdlr (NM_010700.3)* (NPVY to AAVA)]) were produced and inserted into lentiviral packaging vectors. Packaged viruses were collected from packaging cells and further amplified. Lentiviruses containing control, WT Ldlr, and mutated Ldlr vectors were transfected into oligodendrocytes or injected into the CC using stereotaxic injection. Lentiviruses carrying each of these plasmids were purchased from Cyagen Biosciences Inc. As for stereotaxic injection, after deeply anesthetizing with isoflurane, WT or $\mathrm{Ldlr}^{-/}$mice were placed into a stereotactic frame. A hole was drilled in the skull to inject $4 \mu \mathrm{L}$ of the lentivirus solution or $3 \mu \mathrm{L}$ suspensions of 200,000 prelabeled OPCs in phosphate-buffered saline (PBS) into the CC $(0.5 \mathrm{~mm}$ anterior-posterior, $1.0 \mathrm{~mm}$ medial-lateral, $-2.1 \mathrm{~mm}$ dorsal-ventral relative to bregma).

Histological analyses. In brief, $18-\mu \mathrm{m}$-thick brain tissue sections or oligodendrocyte coverslips were fixed in $4 \%$ paraformaldehyde for 10 minutes at room temperature before immunolabeling, followed by blocking in $10 \%$ goat serum, $1 \%$ bovine serum albumin (BSA), and $0.1 \%$ Triton X-100 in PBS at room temperature $(49,50)$. The sections were then incubated with primary antibodies overnight at $4^{\circ} \mathrm{C}$. Signals were detected by incubating the sections with appropriate fluorophore-conjugated secondary antibody. Luxol fast blue or Black-Gold II staining (MilliporeSigma) was used to evaluate myelin integrity. The severity of white matter lesions was scored as previously described (42) or assessed by the percentage of myelinated area (51). TUNEL assays (Roche Applied Science or Beyotime) were performed on brain sections according to the manufacturer's manual as previously described (46, 52). LDL uptake assay (ab133127, Abcam) was conducted in viable cells in accordance with the manufacturer's protocol. Photographs were captured with a confocal laser scanning microscope. Combined FISH for miR-344e-3p and immunofluorescent labeling for $\mathrm{CC} 1$ were performed. We used the double-FAM-labeled miR-344e-3p probes to detect miR-344e-3p and anti-CC1 antibody to locate mature oligodendrocytes. Nuclei were stained with DAPI and the slides were mounted in anti-fade reagent with DAPI (Vector Laboratories).

Coimmunoprecipitation, GST pull-down assay, and immunoblotting. Total cell lysates from the CC or oligodendrocytes were extracted and prepared by using RIPA lysis buffer (Cell Signaling Technology) containing 1\% PMSF on ice. Sample protein concentration was determined by BCA assay (Beyotime).

For coimmunoprecipitation, protein extracts were incubated with goat anti-LDLR (sc-11824, Santa Cruz Biotechnology) or control IgG from the same species overnight at $4^{\circ} \mathrm{C}$. The immune complexes were then bound to Protein A or G conjugated to microbeads (Cell Signaling Technology). After the supernatant was removed by centrifugation, eluted proteins were processed for immunoblotting analysis.

For GST pull-down experiments, purified PTB (Shc 146-317)-GST fusion proteins were incubated with 2 LDLR fragments conjugated with HIS (WT: LDLR-NPVY 760-862; mutated: LDLR-AAVA 760862), which were synthesized by GeneCreate Biological Engineering Co., Ltd. The proteins were then bound to glutathione-sepharose beads. Eluted proteins were analyzed by immunoblotting.

Immunoblotting was performed as described previously (53). Equal amounts of protein were used for SDS-PAGE and specific protein signals were visualized by chemiluminescence using the ECL Western blotting detection system (MilliporeSigma).

miRNA sequencing, serum miRNA extraction, and real-time PCR. Total RNA was extracted from the CC of control, 4-week BCAS, and 8 -week BCAS mice ( $n=4$ /group). miRNA sequencing was performed using the Illumina HiSeq 2500 platform and data analysis was performed by Ribo Bio. Serum miRNAs were extracted from patients using the miRNeasy Serum/Plasma kit (QIAGEN) according to the manufacturer's manual. Equal amounts of C. elegans miR-39-3p miRNA (miRNeasy Serum/Plasma Spike-In Control, QIAGEN) were added to each sample. Total RNA was extracted from brain tissue or cultured cells using TRIzol (Invitrogen) followed by cDNA synthesis by a RevertAid First-Strand cDNA Synthesis Kit (Thermo Fisher Scientific). TaqMan probe- or SYBR Green-based quantitative PCR was conducted 
on a Stratagene Mx3000P real-time PCR system (Agilent Technologies). Specific stem-loop primers were used for reverse transcription of miRNAs (Supplemental Table 4). The primer pairs are listed in Supplemental Table 5.

miRNA transfection and dual-luciferase reporter assay. For miRNA transfection, 293T cells or OPCs were incubated with $50 \mathrm{nM}$ miR344e/410-3p mimic/agomir or antagomir using Lipofectamine 3000 Reagent (Thermo Fisher Scientific). An equal concentration of a nontargeting control sequence was added to experimental samples as controls for non-sequence-specific effects in miRNA experiments.

The entire and mutated $3^{\prime}$ UTR of human and mouse LDLR were generated by Ribo Bio. 293T cells were plated into 96-well plates and cotransfected with $1 \mu \mathrm{g}$ of the indicated $3^{\prime}$ UTR luciferase reporter vectors and $100 \mathrm{nM}$ miR-344e/410-3p mimics or control mimic (Ribo Bio). Renilla and firefly luciferase activities were measured using the Dual-Luciferase Reporter Assay System (E1910, Promega) with the GloMax-Multi+ Detection System (Promega). Experiments were performed in duplicate wells and repeated 4 times.

$E M$. Mice were euthanized and the CC was isolated and fixed in $2.5 \%$ glutaraldehyde and processed as previously reported (54). Photographs were obtained using a HITACHI TEM system. The percentage of myelinated axons and myelin thickness were calculated and analyzed.

Image acquisition and fiber tracking. A diagnosis of leukoaraiosis was based on brain MR imaging features with a 3.0 T MR scanner (MAGENTOM Trio 3.0 T, Siemens). Mouse MR data were acquired using a 7 T MRI scanner with a maximum gradient of $360 \mathrm{mT} / \mathrm{m}$ (PharmaScan, Bruker BioSpin). The 3D fiber tracts were created using the Diffusion Toolkit (http://trackvis.org/dtk/) and demonstrated by TrackVis (www.trackvis.org). For details regarding patients and mouse imaging see Supplemental Materials.

Study approval. All experimental protocols were carried out in accordance with NIH guidelines and approved by the Jinling Hospital Animal Care Committee. Written informed consent was obtained from participants or legal representatives for the use of patients' blood before inclusion in the study, and the protocol was approved by the Ethical Committee of Jinling Hospital.

Statistics. Statistical analysis was performed using SPSS software, version 21.0 (SPSS Inc.). All results were scored while blinded to genotype before quantification. Continuous variables are represented as mean $\pm S D$, and were analyzed with unpaired or paired $t$ tests or 1-way ANOVA followed by Tukey's post hoc test, as appropriate. We also evaluated the risk factors of leukoaraiosis by performing binary logistic regression analysis. Multivariable analysis was further adjusted for variables with $P$ less than 0.1 in univariate analysis. Variable selection was carried out by a random-forest model based on the presence/absence of leukoaraiosis (R statistical environment, version 3.5.1). Statistical significance was established at $P$ less than 0.05 .

\section{Author contributions}

$\mathrm{XL}$ and RY conceptualized and supervised the study. RY and Y Xie designed the research and wrote the whole manuscript. Y Xie, XZ, PX, NZ, and YZ performed the animal experiments. Y Xie, PX, $\mathrm{YH}, \mathrm{MP}$, and DC conducted the cellular experiments. Y Xie, XZ, YL, KY, and TW recruited the patients and collected serum with clinical information. Y Xie, XZ, PX, RS, and JC performed serum miRNA extraction and validation. LX, HG, WS, JL, RL, Y Xiong, and DL, who were blinded to the group information, analyzed all the results. YW and GL assisted with breeding and maintaining colonies of knockout rodents.

\section{Acknowledgments}

We thank Mingming Gao and Xin Guo from Peking University and the Key Laboratory of Cardiovascular Sciences for their help in breeding $L d l r$-knockout rodents. This project was supported by grants from the National Natural Science Foundation of China (grant nos. 81701180 to Y Xie, 81901248 to XZ, 81530038 to XL, 81471182 to RY, and 81671172 to Y Xiong), and the China Postdoctoral Science Foundation (grant nos. 2019T120968 and 2019M664011 to Y Xie).

Address correspondence to: Xinfeng Liu or Ruidong Ye, Department of Neurology, Jinling Hospital, Medical School of Nanjing University, No. 305 East Zhongshan RD, Nanjing, Jiangsu 210000, China. Phone: 86.25.84801861; Email: xfliu2@vip.163. com (X. Liu); or yeruid@gmail.com (R. Ye).
1. Dewar D, et al. Oligodendrocytes and ischemic brain injury. J Cereb Blood Flow Metab. 2003;23(3):263-274.

2. Hawkins V, Butt A. TASK-1 channels in oligodendrocytes: a role in ischemia mediated disruption. Neurobiol Dis. 2013;55(100):87-94.

3. Simons M, Nave KA. Oligodendrocytes: myelination and axonal support. Cold Spring Harb Perspect Biol. 2015;8(1):a020479.

4. Caprariello AV, et al. Apoptosis of oligodendrocytes in the central nervous system results in rapid focal demyelination. Ann Neurol. 2012;72(3):395-405.

5. Ahmad AS, et al. Considerations for the optimization of induced white matter injury preclinical models. Front Neurol. 2015;6:172.

6. Wang Y, et al. White matter injury in ischemic stroke. Prog Neurobiol. 2016;141:45-60.

7. Saher G, Stumpf SK. Cholesterol in myelin biogenesis and hypomyelinating disorders. Biochim Biophys Acta . 2015;1851(8):1083-1094.
8. Pietiäinen V, et al. NDRG1 functions in LDL receptor trafficking by regulating endosomal recycling and degradation. J Cell Sci. 2013;126(pt 17):3961-3971.

9. Vuorio AF, Kovanen PT. Do statins reduce the incidence of stroke in familial hypercholesterolemia? Expert Rev Cardiovasc Ther. 2011;9(3):349-353.

10. Schmitz S, et al. White matter brain lesions in midlife familial hypercholesterolemic patients at 3-Tesla magnetic resonance imaging. Acta Radiol. 2008;49(2):184-189.

11. Hyttinen L, et al. White matter hyperintensities on T2-weighted MRI images among DNA-verified older familial hypercholesterolemia patients. Acta Radiol. 2009;50(3):320-326.

12. ten Dam VH, et al. Effect of pravastatin on cerebral infarcts and white matter lesions. Neurology. 2005;64(10):1807-1809.

13. Minkner K, et al. White matter lesions in watershed territories studied with MRI and parenchymography: a comparative study. Neuroradiology.
2005;47(6):425-430

14. Go GW, Mani A. Low-density lipoprotein receptor (LDLR) family orchestrates cholesterol homeostasis. Yale J Biol Med. 2012;85(1):19-28.

15. Safina D, et al. Low-density lipoprotein receptor-related protein 1 is a novel modulator of radial glia stem cell proliferation, survival, and differentiation. Glia. 2016;64(8):1363-1380.

16. Lin L, Hu K. LRP-1: functions, signaling and implications in kidney and other diseases. Int JMol Sci. 2014;15(12):22887-22901.

17. Yatomi Y, et al. Type 2 diabetes reduces the proliferation and survival of oligodendrocyte progenitor cells in ishchemic white matter lesions. Neuroscience. 2015;289:214-223.

18. Miyanohara J, et al. TRPM2 channel aggravates CNS inflammation and cognitive impairment via activation of microglia in chronic cerebral hypoperfusion. J Neurosci. 2018;38(14):3520-3533.

19. Kirby L, et al. Oligodendrocyte precursor cells present antigen and are cytotoxic targets in 
inflammatory demyelination. Nat Commun. 2019;10(1):3887.

20. Zhang Y, et al. An RNA-sequencing transcriptome and splicing database of glia, neurons, and vascular cells of the cerebral cortex. J Neurosci. 2014;34(36):11929-11947.

21. Zhao S, et al. Selective expression of LDLR and VLDLR in myelinating oligodendrocytes. Dev Dyn. 2007;236(9):2708-2712.

22. Diestschy J, Turley S. Cholesterol metabolism in the central nervous system during early development and in the mature animal. J Lipid Res. 2004;45(8):1375-1397.

23. Osono Y, et al. Role of the low density lipoprotein receptor in the flux of cholesterol through the plasma and across the tissues of the mouse. JClin Invest. 1995;95(3):1124-1132.

24. Elder GA, et al. Elevated plasma cholesterol does not affect brain Abeta in mice lacking the low-density lipoprotein receptor. J Neurochem. 2007;102(4):1220-1231

25. Fryer JD, et al. The low density lipoprotein receptor regulates the level of central nervous system human and murine apolipoprotein $\mathrm{E}$ but does not modify amyloid plaque pathology in PDAPP mice. J Biol Chem. 2005;280(27):25754-25759.

26. Quan G, et al. Ontogenesis and regulation of cholesterol metabolism in the central nervous system of the mouse. Brain Res Dev Brain Res. 2003;146(1-2):87-98.

27. Taha AY, et al. Brainstem concentrations of cholesterol are not influenced by genetic ablation of the low-density lipoprotein receptor. Neurochem Res. 2009;34(2):311-315.

28. Wang N, et al. ATP-binding cassette transporter A1 (ABCA1) functions as a cholesterol efflux regulatory protein. J Biol Chem. 2001;276(26):23742-23747.

29. Zhou X, et al. Genetic deletion of low density lipoprotein receptor impairs sterol-induced mouse macrophage ABCA1 expression. A new SREBP1-dependent mechanism. J Biol Chem. 2008;283(4):2129-2138.

30. Chen WJ, et al. NPXY, a sequence often found in cytoplasmic tails, is required for coated pit-mediated internalization of the low density lipoprotein receptor. J Biol Chem.
1990;265(6):3116-3123.

31. Garcia CK, et al. Autosomal recessive hypercholesterolemia caused by mutations in a putative LDL receptor adaptor protein. Science. 2001;292(5520):1394-1398.

32. Beglova N, Blacklow SC. The LDL receptor: how acid pulls the trigger. Trends Biochem Sci. 2005;30(6):309-317.

33. Stockinger $\mathrm{W}$, et al. The PX-domain protein SNX17 interacts with members of the LDL receptor family and modulates endocytosis of the LDL receptor. EMBO J. 2002;21(16):4259-4267.

34. Guillaumond F, et al. Cholesterol uptake disruption, in association with chemotherapy, is a promising combined metabolic therapy for pancreatic adenocarcinoma. Proc Natl Acad Sci U S A. 2015;112(8):2473-2478.

35. Zhao LJ, et al. Hepatitis C virus E2 protein promotes human hepatoma cell proliferation through the MAPK/ERK signaling pathway via cellular receptors. Exp Cell Res. 2005;305(1):23-32.

36. Zhao LJ, et al. Up-regulation of ERK and p38 MAPK signaling pathways by hepatitis $\mathrm{C}$ virus $\mathrm{E} 2$ envelope protein in human $\mathrm{T}$ lymphoma cell line. J Leukoc Biol. 2006;80(2):424-432.

37. Asmussen JW, et al. Peptides modeled after the alpha-domain of metallothionein induce neurite outgrowth and promote survival of cerebellar granule neurons. Eur JCell Biol. 2009;88(8):433-443.

38. Ishii A, et al. ERK1/ERK2 MAPK signaling is required to increase myelin thickness independent of oligodendrocyte differentiation and initiation of myelination. J Neurosci. 2012;32(26):8855-8864.

39. Ishii A, et al. Sustained activation of ERK1/2 MAPK in oligodendrocytes and schwann cells enhances myelin growth and stimulates oligodendrocyte progenitor expansion. J Neurosci. 2013;33(1):175-186

40. Michel K, et al. Translational control of myelin basic protein expression by ERK2 MAP kinase regulates timely remyelination in the adult brain. J Neurosci. 2015;35(20):7850-7865.

41. Hossain S, et al. Regulation of periphera myelination by Src-like kinases. Exp Neurol. 2010;226(1):47-57.

42. Shibata M, et al. White matter lesions and glial activation in a novel mouse model of chronic cerebral hypoperfusion. Stroke. 2004;35(11):2598-2603.

43. Arai K, Lo EH. An oligovascular niche: cerebral endothelial cells promote the survival and proliferation of oligodendrocyte precursor cells. J Neurosci. 2009;29(14):4351-4355.

44. Patel JR, et al. CXCR4 promotes differentiation of oligodendrocyte progenitors and remyelination. Proc Natl Acad Sci U S A. 2010;107(24):11062-11067.

45. Chen $\mathrm{Y}$, et al. Isolation and culture of rat and mouse oligodendrocyte precursor cells. Nat Protoc. 2007;2(5):1044-1051.

46. Xu P, et al. Breast cancer susceptibility protein 1 (BRCA1) rescues neurons from cerebral ischemia/reperfusion injury through NRF2-mediated antioxidant pathway. Redox Biol. 2018;18:158-172.

47. Gardner A, et al. Myelination of rodent hippocampal neurons in culture. Nat Protoc 2012;7(10):1774-1782

48. Deshmukh VA, et al. A regenerative approach to the treatment of multiple sclerosis. Nature. 2013;502(7471):327-332.

49. Xie Y, et al. Human albumin attenuates excessive innate immunity via inhibition of microglial Mincle/Syk signaling in subarachnoid hemorrhage. Brain Behav Immun. 2017;60:346-360.

50. Xie Y, et al. Human albumin improves long-term behavioral sequelae after subarachnoid hemorrhage through neurovascular remodeling. Crit Care Med. 2015;43(10):e440-e449.

51. Chen T, et al. Butyrate suppresses demyelination and enhances remyelination. J Neuroinflammation. 2019;16(1):165.

52. Ye R, et al. Ginsenoside Rd attenuates mitochondrial dysfunction and sequential apoptosis after transient focal ischemia. Neuroscience. 2011;178:169-180.

53. Ye R, et al. Sevoflurane preconditioning improves mitochondrial function and long-term neurologic sequelae after transient cerebral ischemia: role of mitochondrial permeability transition. Crit Care Med. 2012;40(9):2685-2693.

54. Camargo N, et al. Oligodendroglial myelination requires astrocyte-derived lipids. PLoS Biol. 2017;15(5):e1002605. 